\title{
LOWER BOUNDS ON THE NUMBER OF RATIONAL POINTS OF JACOBIANS OVER FINITE FIELDS AND APPLICATION TO ALGEBRAIC FUNCTION FIELDS IN TOWERS
}

\author{
S. BALLET, R. ROLLAND, AND S. TUTDERE
}

\begin{abstract}
We give effective bounds for the class number of any algebraic function field of genus $g$ defined over a finite field. These bounds depend on the possibly partial information on the number of places on each degree $\leq g$. Such bounds are especially useful for estimating the class number of function fields in towers of function fields over finite fields. We give examples in the case of asymptotically good towers. In particular we estimate the class number of function fields which are steps of towers having one or several positive Tsfasman-Vladut invariants. Note that the study is not done asymptotically, but for each individual step of the towers for which we determine precise parameters .
\end{abstract}

\section{Introduction}

1.1. General context. We recall that the class number $h\left(F / \mathbb{F}_{q}\right)$ of an algebraic function field $F / \mathbb{F}_{q}$ defined over a finite field $\mathbb{F}_{q}$ is the cardinality of the Picard group of $F / \mathbb{F}_{q}$. This numerical invariant corresponds to the number of $\mathbb{F}_{q}$-rational points of the Jacobian of any curve $X\left(\mathbb{F}_{q}\right)$ having $F / \mathbb{F}_{q}$ as algebraic function field. Estimating the class number of an algebraic function field is a classical problem. By the standard estimates deduced from the results of Weil [20] 21], we know that

$$
(\sqrt{q}-1)^{2 g} \leq h\left(F / \mathbb{F}_{q}\right) \leq(\sqrt{q}+1)^{2 g},
$$

where $g$ is the genus of $F / \mathbb{F}_{q}$. Moreover, these estimates hold for any Abelian variety. The value of $h\left(F / \mathbb{F}_{q}\right)$ is completely determined by the zeta function of the function field. But generally, we have not enough information to compute the zeta function effectively. Finding good estimates for the class number $h\left(F / \mathbb{F}_{q}\right)$ from a partial information on the number of places of degrees $\leq g-1$ is a difficult problem. For $g=1$, namely for elliptic curves, the class number is the number of $\mathbb{F}_{q}$-rational points of the curve and this case has been extensively studied. So, from now on we assume that $g \geq 2$. In [11], Lachaud and Martin-Deschamps proved that $h\left(F / \mathbb{F}_{q} \geq h_{L M D}\right.$ where

$$
h_{L M D}=q^{g-1} \frac{(q-1)^{2}}{(q+1)(g+1)} .
$$

This estimate do no use any information on the number of places. Recently in [4], the two first authors gave the following results:

Date: June 11, 2018.

2010 Mathematics Subject Classification. Primary 14H05; Secondary 12E20.

Key words and phrases. finite field, Jacobian, algebraic function field, class number, tower. 
Theorem 1.1. Let $F / \mathbb{F}_{q}$ be an algebraic function field defined over $\mathbb{F}_{q}$ of genus $g \geq 2$ and $h$ the class number of $F / \mathbb{F}_{q}$. Suppose that the numbers $B_{1}$ and $B_{r}$ of places of degree respectively 1 and $r$ are such that $B_{1} \geq 1$ and $B_{r} \geq 1$. Let us denote by $K_{1}(i, B)$ and $K_{2}(q, j, B)$ the following numbers:

$$
K_{1}(i, B)=\left(\begin{array}{c}
B+i \\
B
\end{array}\right) \quad \text { and } \quad K_{2}(q, j, B)=\sum_{i=0}^{j} \frac{1}{q^{i}}\left(\begin{array}{c}
B+i-1 \\
B-1
\end{array}\right) .
$$

Then, the following inequality holds:

$$
h \geq h_{B R}
$$

where

$$
\begin{aligned}
& h_{B R}=\frac{(q-1)^{2}}{(g+1)(q+1)-B_{1}}\left[K_{2}\left(q, r-1, B_{1}\right) q^{g-1} \sum_{m=0}^{\left\lfloor\frac{g-2}{r}\right\rfloor-1} \frac{1}{\left(q^{r}\right)^{m}}\left(\begin{array}{c}
B_{r}+m-1 \\
B_{r}-1
\end{array}\right)\right. \\
& +q K_{2}\left(q,(g-2) \bmod r, B_{1}\right)\left(\begin{array}{c}
B_{r}+\left\lfloor\frac{g-2}{r}\right\rfloor-1 \\
B_{r}-1
\end{array}\right) \\
& +K_{1}\left(r-1, B_{1}\right)\left(\begin{array}{c}
B_{r}+\left\lfloor\frac{g-1}{r}\right\rfloor-1 \\
B_{r}^{r}
\end{array}\right) \\
& \left.+K_{1}\left((g-2) \bmod r, B_{1}\right)\left(\begin{array}{c}
B_{r}+\left\lfloor\frac{g-1}{r}\right\rfloor-1 \\
B_{r}-1
\end{array}\right)\right]
\end{aligned}
$$

Theorem 1.2. Let $F / \mathbb{F}_{q}$ be a function field of genus $g \geq 2$ defined over a finite field $\mathbb{F}_{q}$ and $h$ be its the class number. Suppose that the numbers $B_{1}$ and $B_{r}$ of places of degree respectively 1 and $r$ are such that $B_{1}>0$ and $B_{r}>0$ for any $r \in \mathbb{N}$. Let $m_{r}(n)$ be the quotient of the Euclidean division of the integer $n$ by the integer $r$. Then

$$
h \geq \frac{(q-1)^{2}}{(g+1)(q+1)} q^{g-1} h_{r}
$$

where $h_{r}$ is defined as follows:

(1) For any $B_{r}$, let us set

$$
f_{r}= \begin{cases}0 & \text { if } \quad \frac{g-2}{2}<r \leq g-2 \\ 1 & \text { if } r \leq \frac{g-2}{2} \text { and } B_{r}<q^{r} \\ \min \left(\left\lfloor\frac{B_{r}-q^{r}}{q^{r}-1}\right\rfloor+1, m_{r}(g-2)-1\right) & \text { if } r \leq \frac{g-2}{2} \text { and } B_{r} \geq q^{r} .\end{cases}
$$

then

$$
h_{r}=\left(\frac{q^{r m_{r}(g-2)}-1}{q^{r\left(m_{r}(g-2)-1\right)}\left(q^{r}-1\right)}+\frac{\left(B_{r}-1\right)}{q^{r}} f_{r}\right) .
$$

(2) If $B_{r} \leq m_{r}(g-2)$ then

$$
h_{r}=\left(\frac{q^{r}}{q^{r}-1}\right)^{B_{r}-1}
$$

(3) If $B_{r}>m_{r}(g-2)$ and $r \leq \frac{g-2}{2}$ then

$$
h_{r}=\left(1+\frac{B_{r}}{q^{r}\left(m_{r}(g-2)-1\right)}\right)^{m_{r}(g-2)-1} .
$$


(4) If $B_{r}+1 \leq\left(m_{r}(g-2)-1\right)\left(q^{r}-1\right)$ then

$$
h_{r}=\left[\left(\frac{q^{r}}{q^{r}-1}\right)^{B_{r}}-B_{r}\left(\begin{array}{c}
B_{r}+m_{r}(g-2)-1 \\
B_{r}
\end{array}\right)\left(\frac{1}{q^{r}}\right)^{m_{r}(g-2)}\right] .
$$

Note that the different cases for the value $h_{r}$ are not mutually exclusive, no case is better than the others in absolute terms. In fact, the optimal choice of $h_{r}$ depends on the parameters (curve, genus, places of degree $r$ etc.). In particular, the assertion (a) is always valid but the estimation can be improved by the other assertions depending on the values of parameters.

However, in all the cases, the above result significantly improves the lower bounds of Lachaud - Martin-Deschamps in [11]. Moreover, they obtain the following asymptotic result [3] which reaches some asymptotics for the Jacobian obtained by Tsfasman [15, Corollary 2] and Tsfasman-Vladut [16] (cf.also [18]):

Theorem 1.3. Let $\mathcal{F} / \mathbb{F}_{q}=\left(F_{k} / \mathbb{F}_{q}\right)_{k \geq 0}$ be a sequence of function fields over a finite field $\mathbb{F}_{q}$ and $\mathcal{G} / \mathbb{F}_{q^{r}}=\left(G_{k} / \mathbb{F}_{q^{r}}\right)_{k \geq 0}$ with $G_{k}=F_{k} \mathbb{F}_{q^{r}}$ the constant field extension of $F_{k}$ for some $r \geq 1$. Let $g_{k}$ be the genus, $h\left(F_{k} / \mathbb{F}_{q}\right)$ be the class number, and $B_{i}\left(F_{k} / \mathbb{F}_{q}\right)$ be the number of places of degree $i$ of $F_{k} / \mathbb{F}_{q}$ for any $i, k \geq 0$.

Let further $\alpha$ be a positive real number. Suppose that $B_{1}\left(F_{k} / \mathbb{F}_{q}\right) \geq 1$ for some $k \geq 0$ and there exists an $r \geq 1$ such that

(1) $\liminf _{k \rightarrow \infty} \frac{B_{r}\left(F_{k} / \mathbb{F}_{q}\right)}{g_{k}}>\alpha$ or

(2) $\frac{1}{r} \cdot \liminf _{k \rightarrow \infty} \sum_{i \mid r} \frac{i B_{i}\left(F_{k} / \mathbb{F}_{q}\right)}{g_{k}}>\alpha$.

Then

$$
h\left(F_{k} / \mathbb{F}_{q}\right)>C\left(\left(\frac{q^{r}}{q^{r}-1}\right)^{\alpha} q\right)^{g_{k}}
$$

where $C>0$ is a constant with respect to $k$.

In particular, it happens when they specialize their study to some families of curves having asymptotically a large number of places of degree $r$ for some value of $r$, namely when $\liminf _{g \rightarrow \infty} B_{r}(g) / g>0$. Recently, in [7] the third authors et al. explicitly constructed towers $\mathcal{F} / \mathbb{F}_{q}=\left(F_{k} / \mathbb{F}_{q}\right)_{k \geq 0}$ of curves having asymptotically a large number of places of degree $r_{i}$ for several distinct values of $r_{i}$, namely with several positive Tsfasman-Vladut invariants:

$$
\beta_{r_{i}}(\mathcal{F})=\lim _{k \rightarrow \infty} B_{r_{i}}\left(F_{k} / \mathbb{F}_{q}\right) / g_{k}>0 .
$$

Consequently, we are interested by estimating the class number of these new towers of algebraic function fields defined over $\mathbb{F}_{q}$, which is the main motivation of this paper. Note that the existence of such towers had already been mentioned by Lebacque et al. [12 but it follows from the class field theory which does not give explicit constructions. Moreover, Lebacque [12, Theorem 7] obtains an explicit version of the Generalized Brauer-Siegel Theorem which is valid in the case of smooth absolutely irreducible Abelian varieties defined over a finite field and for the number fields under the Generalized Riemann Hypothesis(GRH). Specialized to the case of smooth absolutely irreducible curves over finite fields, this theorem leads to the following result:

Theorem 1.4. For any smooth absolutely irreducible curve $X$ of genus $g$ defined over the finite field $\mathbb{F}_{r}$, one has, as $N \rightarrow \infty$ : 


$$
\sum_{m=1}^{N} \mathbb{P} h i_{r^{m}} \log \left(\frac{r^{m}}{r^{m}-1}\right)=\log N+\gamma+\log \left(\aleph_{X} \log r\right)+\mathcal{O}\left(\frac{1}{N}\right)+g \mathcal{O}\left(\frac{r^{-N / 2}}{N}\right)
$$

where $\mathbb{P} h i_{r^{m}}=\#\{\mathfrak{p} \in|X| \mid \operatorname{deg}(\mathfrak{p})=m\},|X|$ denotes the set of closed points of $X$ and $\aleph_{X}$ denotes the residue at $s=1$ of the zeta function $\zeta_{X}$ of $X$. Moreover, the $\mathcal{O}$ constants are effective and do not depend on $X$.

Passing to the limit in the previous result gives the asymptotics of TsfasmanVladut [16] [17]. Note also that as the constants are effective, this result could lead to effective non-asymptotic lower bounds of the class number $h$. However, it is clearly not obvious to obtain these effective bounds. On the other hand, very recently Aubry, Haloui, and Lachaud [1, Proposition 2.1 and Theorem 2.4] (cf. also [2]), in the general context of Abelian Varieties, deduced new lower bounds on the number of points of Jacobians. in particular, they proved the following:

Theorem 1.5. Let $C$ be a curve of genus $g \geq 2$ over $\mathbb{F}_{q}$ with $N$ rational points. Let $J_{C}\left(\mathbb{F}_{q}\right)$ be the Jacobian of $C$ over $\mathbb{F}_{q}$. Then the following inequalities hold:

(I)

$$
\left|J_{C}\left(\mathbb{F}_{q}\right)\right| \geq M(q)^{g}\left(q+1+\frac{N-(q+1)}{g}\right)^{g}
$$

$$
\begin{aligned}
& \text { with } \left.M(q)=\frac{1}{S(h(q))} \text { where } S(h)=\frac{h^{1 / h-1}}{e \log h^{1 / h-1}} \text { (and } S(1)=1\right) \text { denotes the } \\
& \text { Specht's ratio and } h(q)=\left(\begin{array}{c}
q^{1 / 2}+1 \\
q^{1 / 2}-1
\end{array}\right)^{2} \text {. }
\end{aligned}
$$

$$
\left|J_{C}\left(\mathbb{F}_{q}\right)\right| \geq \frac{q-1}{q^{g}-1}\left[\left(\begin{array}{c}
N+2 g-2 \\
2 g-1
\end{array}\right)+\sum_{i=2}^{2 g-1} B_{i}\left(\begin{array}{c}
N+2 g-2-i \\
2 g-1-i
\end{array}\right)\right]
$$

(III) If $N \geq g\left(q^{\frac{1}{2}}-1\right)+1$ then

$$
\left|J_{C}\left(\mathbb{F}_{q}\right)\right| \geq\left(\begin{array}{c}
N+g-1 \\
g
\end{array}\right)-q\left(\begin{array}{c}
N+g-3 \\
g-2
\end{array}\right)
$$

$$
\left|J_{C}\left(\mathbb{F}_{q}\right)\right| \geq \frac{(q-1)^{2}}{(g+1)(q+1)-N}\left[\left(\begin{array}{c}
N+g-2 \\
g-2
\end{array}\right)+\sum_{i=0}^{g-1} q^{g-1-i}\left(\begin{array}{c}
N+i-1 \\
i
\end{array}\right)\right]
$$

Note that Bound (IV) is exactly the particular case of [5, Theorem 3.1] (cf. also Theorem 1.1) obtained with $r=1$.

Let us denote by $h_{A H L}$ the bound (III) which is the most accurate of these bounds with the exception of bound (IV) already known as previously noted (bound $h_{B R}$ ):

$$
h_{A H L}=\left(\begin{array}{c}
N+g-1 \\
g
\end{array}\right)-q\left(\begin{array}{c}
N+g-3 \\
g-2
\end{array}\right) .
$$

Note that, Bound (II) gives no significant result mainly because of the factor $q^{g}$ in denominator. 
1.2. New results. We remark that Theorem 1.1 in the non-asymptotic case and Theorem 1.3 in the asymptotic case are particularly suitable for the curves having many points of a given degree $r$. Here, we are interested in studying curves having for several fixed distinct degrees a lot of points. In this paper we give bounds on the class number of an algebraic function field of one variable defined over the finite field $\mathbb{F}_{q}$ in the non-asymptotic case, namely when the function field is fixed. More precisely, these bounds which are effective bounds depend on possibly partial information on the genus $g$ and the number of places of each degree $\leq g$. In this context, we generalize Theorem 1.1 to obtain more precise bounds taking into account the number of places of several distinct degrees $r_{1}, r_{2}, \ldots, r_{u}$. In particular, we obtain:

Theorem 1.6. Let $F / \mathbb{F}_{q}$ be an algebraic function field defined over $\mathbb{F}_{q}$ of genus $g \geq 2$ and $h$ its class number. For any $r$ such that $1 \leq r \leq g-1$, let $B_{r}$ be the number of places of degree $r$ of $F / \mathbb{F}_{q}$.

For any set $D_{1}$, any set $D_{2}$, any finite set of integers $l=\left\{l_{r}\right\}_{r \in D_{1}}$ and any finite set of integers $m=\left\{m_{r}\right\}_{r \in D_{2}}$ such that

(1) $D_{1} \subseteq\{1, \cdots, g-1\}$;

(2) for any $r \in D_{1}$ we have $B_{r} \geq 1$;

(3) $D_{2} \subseteq\{1, \cdots, g-2\}$;

(4) for any $r \in D_{2}$ we have $B_{r} \geq 1$;

(5) $l_{r} \geq 0, \quad \sum_{r \in D_{1}} r l_{r} \leq g-1$;

(6) $m_{r} \geq 0, \quad \sum_{r \in D_{2}} r m_{r} \leq g-2$.

Then $h \geq h_{B R T}$ where

$$
\begin{array}{r}
h_{B R T}=\frac{(q-1)^{2}}{(g+1)(q+1)-B_{1}}\left(\prod_{r \in D_{1}}\left(\begin{array}{c}
B_{r}+l_{r} \\
l_{r}
\end{array}\right)\right. \\
\left.+q^{g-1} \prod_{r \in D_{2}}\left[\left(\frac{q^{r}}{q^{r}-1}\right)^{B_{r}}-B_{r}\left(\begin{array}{c}
B_{r}+m_{r} \\
B_{r}
\end{array}\right) \int_{0}^{\frac{1}{q^{r}}} \frac{\left(\frac{1}{q^{r}}-t\right)^{m_{r}}}{(1-t)^{B_{r}+m_{r}+1}} \mathrm{dt} .\right]\right) .
\end{array}
$$

We then apply these bounds to each algebraic function field $F_{k} / \mathbb{F}_{q}$ of genus $g_{k}$ of towers having one or several strictly positive Tsfasman-Vladut invariants, namely for which certain ratios $\frac{B_{r_{i}}(k)}{g_{k}}$ have a strictly positive limit when the integer $k$ tends to the infinity, where $B_{r_{i}}(k)$ denotes the number of places of degree $r_{i}$ of $F_{k}$. Note that the study is not done asymptotically, but for each individual step of the towers for which we determine precise parameters: in particular estimations of genus and of number of places of certain degrees for each step. Moreover, we design new examples of compositum towers (built from known asymptotically good towers). Then, we make a precise study of each step of these towers, included the case of known asymptotically good towers. All the given examples are chosen in order to cover a large spectrum of different significant cases. In particular, we construct a basis of examples of towers of algebraic function fields illustrating a variety of significant situations. Moreover, for each step of these towers, we give information about parameters of concerned function field and we show how to best estimate the class numbers depending on the nature of the estimation of parameters (exact value, upper and lower bounds, the presence or absence of rational places).

1.3. Organization of the paper. In Section 2, we fix the main objective in connection with the fundamental equality (4). In Section 3 , we estimate the two terms 
$\Sigma_{1}=\sum_{n=0}^{g-1} A_{n}$ and $\Sigma_{2}=\sum_{n=0}^{g-2} q^{g-1-n} A_{n}$ of the number $S\left(F / \mathbb{F}_{q}\right)$ introduced in Subsection 2 This yields in Section 4 new lower and upper bounds on the class number. In Section 5, we study lower bounds of the class number of each step of several towers of algebraic function fields, in particular we give some numerical estimations.

\section{Preliminaries}

Throughout this paper we use basic facts and notations as in [14]. We consider a function field $F / \mathbb{F}_{q}$ over the finite field with $q$ elements, of genus $g=g(F) \geq 2$. Let $A_{n}=A_{n}\left(F / \mathbb{F}_{q}\right)$ be the number of effective divisors of degree $n$ of $F / \mathbb{F}_{q}$ and $h=h\left(F / \mathbb{F}_{q}\right)$ the class number of $F / \mathbb{F}_{q}$. Let $B_{n}=B_{n}\left(F / \mathbb{F}_{q}\right)$ the number of places of degree $n$ of $F / \mathbb{F}_{q}$.

Let us set

$$
S\left(F / \mathbb{F}_{q}\right)=\sum_{n=0}^{g-1} A_{n}+\sum_{n=0}^{g-2} q^{g-1-n} A_{n} \quad \text { and } \quad R\left(F / \mathbb{F}_{q}\right)=\sum_{i=1}^{g} \frac{1}{\left|1-\pi_{i}\right|^{2}},
$$

where $\left(\pi_{i}, \overline{\pi_{i}}\right)_{1 \leq i \leq g}$ are the reciprocal roots of the numerator of the zeta-function $Z\left(F / \mathbb{F}_{q}, T\right)$ of $F / \mathbb{F}_{q}$. By a result due to G. Lachaud and M. Martin-Deschamps [11, we know that

$$
S\left(F / \mathbb{F}_{q}\right)=h R\left(F / \mathbb{F}_{q}\right) .
$$

Therefore, in order to find good lower bounds on $h$, one just needs to find a good lower bound on $S\left(F / \mathbb{F}_{q}\right)$ and a good upper bound on $R\left(F / \mathbb{F}_{q}\right)$.

It is known by [11] that the quantity $R\left(F / \mathbb{F}_{q}\right)$ is bounded by the following upper bound:

$$
R\left(F / \mathbb{F}_{q}\right) \leq \frac{1}{(q-1)^{2}}\left((g+1)(q+1)-B_{1}\left(F / \mathbb{F}_{q}\right)\right) .
$$

The inequality (5) is obtained as follows:

$$
R\left(F / \mathbb{F}_{q}\right)=\sum_{i=1}^{g} \frac{1}{\left(1-\pi_{i}\right)\left(1-\overline{\pi_{i}}\right)}=\sum_{i=1}^{g} \frac{1}{1+q-\left(\pi_{i}+\overline{\pi_{i}}\right)} .
$$

Multiplying the denominators by the corresponding conjugated quantities, we get:

$$
R\left(F / \mathbb{F}_{q}\right) \leq \frac{1}{(q-1)^{2}} \sum_{i=1}^{g}\left(1+q+\pi_{i}+\overline{\pi_{i}}\right) .
$$

This last inequality associated to the following formula deduced from the Weil's formulas:

$$
\sum_{i=1}^{g}\left(\pi_{i}+\overline{\pi_{i}}\right)=1+q-B_{1}\left(F / \mathbb{F}_{q}\right)
$$

gives the inequality (5). The inequality (5) cannot be improved in the general case. Remark that in the same way we can prove that

$$
R\left(F / \mathbb{F}_{q}\right) \geq \frac{1}{(q+1)^{2}}\left((g+1)(q+1)-B_{1}\left(F / \mathbb{F}_{q}\right)\right) .
$$

Hence, in this paper, we propose to study some lower bounds on $S\left(F / \mathbb{F}_{q}\right)$. In this aim, we determine some bounds of the quantities $\sum_{1}=\sum_{n=0}^{g-1} A_{n}$ and $\sum_{2}=$ 
$\sum_{n=0}^{g-2} q^{g-1-n} A_{n}$ obtained from the number of effective divisors of degree $n \leq g-1$ containing in their support places of some fixed distinct degrees $r_{1}, r_{2}, \ldots, r_{k} \geq 1$. We deduce bounds on the class number.

\section{General Bounds on the sums $\Sigma_{1}$ And $\Sigma_{2}$}

In this section, from an exact formula, giving the number of effective divisors of degree n, we derive an exact formula for $S\left(F / \mathbb{F}_{q}\right)$. Unfortunately this exact formula involves some data, which are not necessarily available. So, we propose methods adapted to cases where some data are not known. These methods do not give longer exact values of $h$ but only more or less accurate estimates.

3.1. The basic equations. In this section, we define the main quantities required for the study of the class number. We set

$$
\Sigma_{1}=\sum_{n=0}^{g-1} A_{n} \quad \text { and } \quad \Sigma_{2}=q^{g-1} \sum_{n=0}^{g-2} \frac{A_{n}}{q^{n}} .
$$

Then we can write

$$
S=\Sigma_{1}+\Sigma_{2}
$$

Let us introduce the following notations that will be used in the whole paper.

$$
\begin{gathered}
\Delta_{1}=\left\{r \mid 1 \leq r \leq g-1 \text { and } B_{r} \geq 1\right\}, \\
\Delta_{2}=\left\{r \mid 1 \leq r \leq g-2 \text { and } B_{r} \geq 1\right\}, \\
\Delta_{1}^{\prime}=\left\{r \mid 2 \leq r \leq g-1 \text { and } B_{r} \geq 1\right\}=\Delta_{1} \backslash\{1\}, \\
\Delta_{2}^{\prime}=\left\{r \mid 2 \leq r \leq g-2 \text { and } B_{r} \geq 1\right\}=\Delta_{2} \backslash\{1\},
\end{gathered}
$$

Let us fix an integer $n \geq 0$ and set

$$
U_{n}=\left\{b=\left(b_{r}\right)_{r \in \Delta_{1}} \mid b_{r} \geq 0 \text { and } \sum_{r \in \Delta_{1}} r b_{r}=n\right\} .
$$

First, note that if $B_{r} \geq 1$ and $b_{r} \geq 0$ in (12), the number of solutions of the equation $n_{1}+n_{2}+\cdots+n_{B_{r}}=b_{r}$ with integers $\geq 0$ is

$$
\left(\begin{array}{c}
B_{r}+b_{r}-1 \\
b_{r}
\end{array}\right)
$$

Then the number of effective divisors of degree $n$ is given by the following result [16] (cf. also [5]):

Proposition 3.1. The number of effective divisors of degree $n$ of an algebraic function field $F / \mathbb{F}_{q}$ is

$$
A_{n}=\sum_{b \in U_{n}}\left[\prod_{r \in \Delta_{1}}\left(\begin{array}{c}
B_{r}+b_{r}-1 \\
b_{r}
\end{array}\right)\right] .
$$

Let $D$ be a subset of $\Delta_{1}$. Let $I(D)=\left(I_{r}\right)_{r \in D}$ be a finite sequence of finite subsets $I_{r}$ of $\mathbb{N}$. We define the hypercube $C_{I(D)}$ as the following product:

$$
C_{I(D)}=\prod_{r \in D} I_{r}
$$

The components of an element $b \in C_{I}(D)$ will be denoted by $b_{r}$, namely $b=\left(b_{r}\right)_{r \in D}$. 
Lemma 3.2. Let $D$ be a subset of $\Delta_{1}$ and $f$ a map from $D \times \mathbb{N}$ to $\mathbb{N}$. Then

$$
\sum_{b \in C_{I}(D)}\left[\prod_{r \in D} f\left(r, b_{r}\right)\right]=\prod_{r \in D}\left[\sum_{b_{r} \in I_{r}} f\left(r, b_{r}\right) .\right]
$$

Proof. Denote by $s$ the cardinality of $D$ and denote by $f_{r}$ the function defined by $f_{r}\left(b_{r}\right)=f\left(r, b_{r}\right)$. Remark that the previous equation can be written:

$$
\begin{gathered}
\sum_{b_{r_{1}} \in I_{r_{1}}} \sum_{b_{r_{2}} \in I_{r_{2}}} \cdots \sum_{b_{r_{s}} \in I_{r_{s}}} f_{r_{1}}\left(b_{r_{1}}\right) f_{r_{2}}\left(b_{r_{2}}\right) \cdots f_{r_{s}}\left(b_{r_{s}}\right)= \\
\left(\sum_{b_{r_{1}} \in I_{r_{1}}} f_{r_{1}}\left(b_{r_{1}}\right)\right)\left(\sum_{b_{r_{2}} \in I_{r_{2}}} f_{r_{2}}\left(b_{r_{2}}\right)\right) \cdots\left(\sum_{b_{r_{s}} \in I_{r_{s}}} f_{r_{s}}\left(b_{r_{s}}\right)\right) .
\end{gathered}
$$

The first member is the development of the product of the second member.

3.2. The sum $\Sigma_{1}$. In this section, we first give an exact formula of the sum $\Sigma_{1}$. We then deduce lower and upper bounds for this sum. We obtain the following from Proposition 3.1 and (7):

\section{Proposition 3.3.}

$$
\Sigma_{1}=\sum_{n=0}^{g-1} A_{n}=\sum_{b \in V_{1}}\left[\prod_{r \in \Delta_{1}}\left(\begin{array}{c}
B_{r}+b_{r}-1 \\
b_{r}
\end{array}\right)\right],
$$

where

$$
V_{1}=\bigcup_{n=0}^{g-1} U_{n}=\left\{b=\left(b_{r}\right)_{r \in \Delta_{1}} \mid b_{r} \geq 0 \text { and } \sum_{r \in \Delta_{1}} r b_{r} \leq g-1\right\},
$$

We can now establish a lower bound for the quantity $\Sigma_{1}$ :

Theorem 3.4 (Lower bound). Let $F / \mathbb{F}_{q}$ be an algebraic function field of genus $g \geq 2$.

(1) Let $m=\left(m_{r}\right)_{r \in \Delta_{1}}$ be a finite sequence of integers $m_{r} \geq 0$ such that $\sum_{r \in \Delta_{1}} r m_{r} \leq g-1$. Then

$$
\Sigma_{1} \geq \prod_{r \in \Delta_{1}}\left(\begin{array}{c}
B_{r}+m_{r} \\
m_{r}
\end{array}\right) .
$$

(2) Suppose that $B_{1} \geq 1$. Let $r_{1}, r_{2}, \cdots, r_{u}$ be distinct elements in $\Delta_{1}^{\prime}$. Then

$$
\begin{aligned}
\Sigma_{1} & \geq\left(\begin{array}{c}
B_{1}+g-1 \\
g-1
\end{array}\right) \\
& +\sum_{i=1}^{u}\left(\left(\begin{array}{c}
B_{r_{i}}+\left\lfloor\frac{g-1}{r_{i}}\right\rfloor \\
\left\lfloor\frac{g-1}{r_{i}}\right\rfloor
\end{array}\right)-1\right)\left(\begin{array}{c}
B_{1}+\left((g-1) \bmod r_{i}\right) \\
(g-1) \bmod r_{i}
\end{array}\right) .
\end{aligned}
$$

Proof.

(1) Let us consider the hypercube $C_{I\left(\Delta_{1}\right)}$ defined by $I\left(\Delta_{1}\right)=\left(I_{r}\right)_{r \in \Delta_{1}}$ where $I_{r}$ is the interval $I_{r}=\left\{0, \cdots, m_{r}\right\}$. The condition $\sum_{r \in \Delta_{1}} r m_{r} \leq g-1$ implies that $C_{I\left(\Delta_{1}\right)} \subseteq V_{1}$. Then by using Proposition 3.3, the following holds:

$$
\Sigma_{1}=\sum_{b \in V_{1}}\left[\prod_{r \in \Delta_{1}}\left(\begin{array}{c}
B_{r}+b_{r}-1 \\
b_{r}
\end{array}\right)\right] \geq \sum_{b \in C_{I\left(\Delta_{1}\right)}}\left[\prod_{r \in \Delta_{1}}\left(\begin{array}{c}
B_{r}+b_{r}-1 \\
b_{r}
\end{array}\right)\right] .
$$


Now, by Lemma 3.2 we get

$$
\Sigma_{1} \geq \prod_{r \in \Delta_{1}}\left[\sum_{b_{r}=0}^{m_{r}}\left(\begin{array}{c}
B_{r}+b_{r}-1 \\
b_{r}
\end{array}\right)\right]=\prod_{r \in \Delta_{1}}\left(\begin{array}{c}
B_{r}+m_{r} \\
m_{r}
\end{array}\right) .
$$

(2) For each $0 \leq i \leq u$, we introduce the hypercube $C_{I_{r}^{(i)}\left(\Delta_{1}\right)}$ as follows:

$$
I_{r}^{(0)}= \begin{cases}\{0, \cdots, g-1\} & \text { if } r=1, \\ \{0\} & \text { if } r>1 .\end{cases}
$$

and for $i>0$,

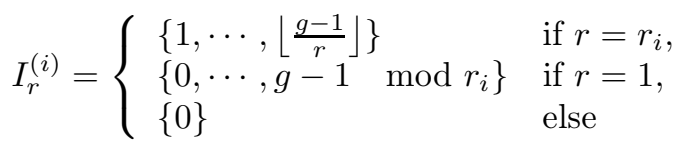

These hypercubes are pairwise disjoint and all are included in $V_{1}$. Then

$$
\begin{aligned}
& \Sigma_{1} \geq \sum_{i=0}^{u} \prod_{r \in \Delta_{1}} \sum_{b_{r} \in I_{r}^{(i)}}\left(\begin{array}{c}
B_{r}+b_{r}-1 \\
b_{r}
\end{array}\right)=\sum_{b_{1}=0}^{g-1}\left(\begin{array}{c}
B_{1}+b_{1}-1 \\
b_{1}
\end{array}\right) \\
& +\sum_{i=1}^{u}\left(\sum_{b_{r_{i}}=1}^{\left\lfloor\frac{g-1}{\left.r_{i}\right\rfloor}\right.}\left(\begin{array}{c}
B_{r_{i}}+b_{r_{i}}-1 \\
b_{r_{i}}
\end{array}\right)\right)\left(\sum_{b_{1}=0}^{(g-1)} \bmod r_{i}\left(\begin{array}{c}
B_{1}+b_{1}-1 \\
b_{1}
\end{array}\right)\right) \\
& =\left(\begin{array}{c}
B_{1}+g-1 \\
g-1
\end{array}\right)
\end{aligned}
$$

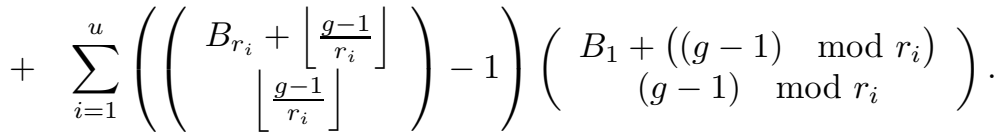

Remark 3.5. By using (18) with $u=1$, we obtain the following:

$$
\begin{aligned}
& \Sigma_{1} \geq\left(\begin{array}{c}
B_{1}+g-1 \\
g-1
\end{array}\right)+\left(\begin{array}{c}
B_{r}+\left\lfloor\frac{g-1}{r_{1}}\right\rfloor \\
\left\lfloor\frac{g-1}{r_{1}}\right\rfloor
\end{array}\right)\left(\begin{array}{c}
B_{1}+\left((g-1) \bmod r_{1}\right) \\
(g-1) \bmod r_{1}
\end{array}\right) \\
& -\left(\begin{array}{c}
B_{1}+\left((g-1) \bmod r_{1}\right) \\
(g-1) \bmod r_{1}
\end{array}\right)
\end{aligned}
$$

which is better than the following formula obtained in [5]:

$$
\Sigma_{1} \geq\left(\begin{array}{c}
B_{r}+\left\lfloor\frac{g-1}{r_{1}}\right\rfloor \\
\left\lfloor\frac{g-1}{r_{1}}\right\rfloor
\end{array}\right)\left(\begin{array}{c}
B_{1}+\left((g-1) \bmod r_{1}\right) \\
(g-1) \bmod r_{1}
\end{array}\right)
$$

except in the case $r_{1}=1$ where the two bounds are the same.

Theorem 3.6 (Upper bound). Let $F / \mathbb{F}_{q}$ be an algebraic function field of genus $g \geq 2$. Then the following holds:

$$
\Sigma_{1} \leq \prod_{r \in \Delta_{1}}\left(\begin{array}{c}
B_{r}+\left\lfloor\frac{g-1}{r}\right\rfloor \\
\left\lfloor\frac{g-1}{r}\right\rfloor
\end{array}\right) .
$$


Proof. Let us consider the hypercube $C_{I\left(\Delta_{1}\right)}$ where $I\left(\Delta_{1}\right)=\left(I_{r}\right)_{r \in \Delta_{1}}$ is such that for any $r \in \Delta_{1}$, the subset $I_{r}$ is $\left\{0, \cdots,\left\lfloor\frac{g-1}{r}\right\rfloor\right\}$. Hence, $V_{1} \subseteq C_{I\left(\Delta_{1}\right)}$ and the following holds:

$$
\Sigma_{1} \leq \prod_{r \in \Delta_{1}}\left[\sum_{b_{r}=0}^{\left\lfloor\frac{g-1}{r}\right\rfloor}\left(\begin{array}{c}
B_{r}+b_{r}-1 \\
b_{r}
\end{array}\right)\right]=\prod_{r \in \Delta_{1}}\left(\begin{array}{c}
B_{r}+\left\lfloor\frac{g-1}{r_{i}}\right\rfloor \\
\left\lfloor\frac{g-1}{r_{i}}\right\rfloor
\end{array}\right)
$$

3.3. The sum $\Sigma_{2}$. In this section, we first give an exact formula of the sum $\Sigma_{2}$. Then, we deduce lower and upper bounds of this sum. From Proposition 3.1 and (7), we obtain:

\section{Proposition 3.7.}

$$
\Sigma_{2}=q^{g-1} \sum_{b \in V_{2}}\left[\prod_{r \in \Delta_{2}} \frac{1}{q^{r b_{r}}}\left(\begin{array}{c}
B_{r}+b_{r}-1 \\
b_{r}
\end{array}\right)\right],
$$

where

$$
V_{2}=\bigcup_{n=0}^{g-2} U_{n}=\left\{b=\left(b_{r}\right)_{r \in \Delta_{2}} \mid \sum_{r \in \Delta_{2}} r b_{r} \leq g-2\right\} .
$$

Theorem 3.8 (Lower bound). Let $F / \mathbb{F}_{q}$ be an algebraic function field of genus $g \geq 2$.

(1) Suppose that $B_{1} \geq 1$. Let $m=\left(m_{r}\right)_{r \in \Delta_{2}}$ be a finite sequence of integers $m_{r} \geq 0$ such that $\sum_{r \in \Delta_{2}} r m_{r} \leq g-2$. Then the following purely multiplicative formula holds:

$$
\Sigma_{2} \geq q^{g-1} \prod_{r \in \Delta_{2}}\left[\sum_{b_{r}=0}^{m_{r}} \frac{1}{q^{r b_{r}}}\left(\begin{array}{c}
B_{r}+b_{r}-1 \\
b_{r}
\end{array}\right)\right] .
$$

(2) Let $r_{1}, r_{2}, \cdots, r_{u}$ be $u$ distinct elements in $\Delta_{2}^{\prime}$. Then the following holds:

$$
\begin{aligned}
& \Sigma_{2} \geq q^{g-1} \sum_{b_{1}=0}^{g-2} \frac{1}{q^{b_{1}}}\left(\begin{array}{c}
B_{1}+b_{1}-1 \\
b_{1}
\end{array}\right)+
\end{aligned}
$$

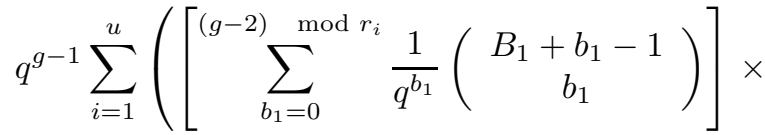

$$
\begin{aligned}
& \left.\left[\sum_{b_{r_{i}}=0}^{\left\lfloor\frac{g-2}{r_{i}}\right\rfloor} \frac{1}{q^{r_{i} b_{r_{i}}}}\left(\begin{array}{c}
B_{r_{i}}+b_{r_{i}}-1 \\
b_{r_{i}}
\end{array}\right)-1\right]\right) .
\end{aligned}
$$

Proof. The proof is similar to that of Theorem 3.4 with the relation $\sum_{r \in \Delta_{2}} r m_{r} \leq$ $g-2$ instead of $\sum_{r \in \Delta_{1}} r m_{r} \leq g-1$. 
Theorem 3.9 (Upper bound). Let $F / \mathbb{F}_{q}$ be an algebraic function field of genus $g \geq 2$. Then

$$
\Sigma_{2} \leq q^{g-1} \prod_{r \in \Delta_{2}}\left[\sum_{b_{r}=0}^{\left\lfloor\frac{g-2}{r}\right\rfloor} \frac{1}{q^{r b_{r}}}\left(\begin{array}{c}
B_{r}+b_{r}-1 \\
b_{r}
\end{array}\right)\right] .
$$

Proof. The proof is similar to that of Theorem 3.6

Remark 3.10. The quantity

$$
Q_{r, s}=\sum_{k=0}^{s} \frac{1}{q^{r k}}\left(\begin{array}{c}
B_{r}+k-1 \\
k
\end{array}\right)
$$

plays an important role in estimating $\Sigma_{2}$. Indeed, by Theorem 3.8 and Theorem 3.9 we have

$$
q^{g-1} \prod_{r \in \Delta_{2}} Q_{r, m_{r}} \leq \Sigma_{2} \leq q^{g-1} \prod_{r \in \Delta_{2}} Q_{r,\left\lfloor\frac{g-2}{r}\right\rfloor}
$$

and

$$
q^{g-1}\left(Q_{1, g-2}+\sum_{i=1}^{u} Q_{r_{i},\left\lfloor\frac{g-2}{r_{i}}\right\rfloor}\right) \leq \Sigma_{2} \leq q^{g-1} \prod_{r \in \Delta_{2}} Q_{r,\left\lfloor\frac{g-2}{r}\right\rfloor} .
$$

Remark 3.11. If $s=0$ then $Q_{r, s}=1$.

The estimation of $Q_{r, s}$ leads to some interesting lower and upper bounds for $\Sigma_{2}$ obtained by injecting the bounds on $Q_{r, s}$ in the formulas of Theorem 3.8

Proposition 3.12. Let $m=\left(m_{r}\right)_{r \in \Delta_{2}}$ be a finite sequence of integers such that $m_{r} \geq 0$ and $\sum_{r \in \Delta_{2}} r m_{r} \leq g-2$. Let $r_{1}, \cdots, r_{u}$ be elements of $\Delta_{2}^{\prime}$. Then the following inequality holds:

$$
\Sigma_{2, \text { inf }} \leq \Sigma_{2} \leq \Sigma_{2, \text { sup }}
$$

where

$$
\Sigma_{2, \text { inf }}=\left\{\begin{array}{l}
q^{g-1} \prod_{r \in \Delta_{2}} Q_{r, m_{r}} \\
\text { or } \\
q^{g-1}\left(Q_{1, g-2}+\sum_{i=1}^{u} Q_{r_{i},\left\lfloor\frac{g-2}{r_{i}}\right\rfloor}\right)
\end{array}\right.
$$

and

$$
\Sigma_{2, \text { sup }}=q^{g-1} \prod_{r \in \Delta_{2}} Q_{r,\left\lfloor\frac{g-2}{r}\right\rfloor} .
$$

Moreover, for any $r \geq 1$ and $s \geq 0$ the following holds:

$$
Q_{r, s}=\left(\frac{q^{r}}{q^{r}-1}\right)^{B_{r}}-B_{r}\left(\begin{array}{c}
B_{r}+s \\
B_{r}
\end{array}\right) \int_{0}^{\frac{1}{q^{r}}} \frac{\left(\frac{1}{q^{r}}-t\right)^{s}}{(1-t)^{B_{r}+s+1}} \mathrm{~d} t .
$$

Proof. We set

$$
\begin{gathered}
S_{r}(X)=\sum_{k=0}^{\infty} X^{k}\left(\begin{array}{c}
B_{r}+k-1 \\
k
\end{array}\right), \quad T_{r}(X, s)=\sum_{k=0}^{s} X^{k}\left(\begin{array}{c}
B_{r}+k-1 \\
k
\end{array}\right), \text { and } \\
R_{r}(X, s)=\sum_{k=s+1}^{\infty} X^{k}\left(\begin{array}{c}
B_{r}+k-1 \\
k
\end{array}\right) .
\end{gathered}
$$


Let us remark that

$$
Q_{r, s}=T_{r}\left(\frac{1}{q^{r}}, s\right) \quad \text { and } \quad S_{r}(X)=\frac{1}{(1-X)^{B_{r}}}
$$

which converges for $|X|<1$ and moreover

$$
S_{r}(X)=T_{r}(X, s)+R_{r}(X, s) .
$$

By the Taylor Formula, we get

$$
R_{r}(X, s)=B_{r}\left(\begin{array}{c}
B_{r}+s \\
B_{r}
\end{array}\right) \int_{0}^{X} \frac{(X-t)^{s}}{(1-t)^{B_{r}+s+1}} \mathrm{~d} t .
$$

Then

$$
Q_{r, s}=\left(\frac{q^{r}}{q^{r}-1}\right)^{B_{r}}-B_{r}\left(\begin{array}{c}
B_{r}+s \\
B_{r}
\end{array}\right) \int_{0}^{\frac{1}{q^{r}}} \frac{\left(\frac{1}{q^{r}}-t\right)^{s}}{(1-t)^{B_{r}+s+1}} \mathrm{~d} t .
$$

Remark 3.13. Note that a lower bound of $\Sigma_{2}$ always can be computed in practice directly from Proposition 3.12 because it is sufficient to know a subset of $\Delta_{1}$ and the corresponding $B_{r}$ (or a lower bound for each corresponding $B_{r}$ ) while the upper bound can not be always computed easily because it need to know precisely the set $\Delta_{1}$ and all the $B_{r}$ of the curve which is difficult when the genus is large for example.

Remark 3.14. Note that if we specialize to the case $r=1$ without knowing information about the other degrees, we obtain $\Sigma_{2, \text { inf }}=q^{g-1} Q_{1, g-2}$ and thus the quantity $\Sigma_{2, \text { inf }}$ is equal to $\Sigma_{2}$ in [5. Formula (2.6)] by Remark 3.10 and Proposition 3.12 .

\section{Bounds ON THE Class NUMBeR}

In this section, using the lower and upper bounds obtained in the previous section, we derive lower ands upper bounds on the class number of an algebraic function field.

Let $F / \mathbb{F}_{q}$ be an algebraic function field defined over $\mathbb{F}_{q}$ of genus $g \geq 2$. For any $r$ such that $1 \leq r \leq g-1$ let $B_{r}$ be the number of places of degree $r$ of $F / \mathbb{F}_{q}$ and $h$ the class number of $F / \mathbb{F}_{q}$.

It is known (cf. [11]) that the class number $h$ is

$$
h=\frac{S\left(F / \mathbb{F}_{q}\right)}{R\left(F / \mathbb{F}_{q}\right)}=\frac{\Sigma_{1}+\Sigma_{2}}{R\left(F / \mathbb{F}_{q}\right)} .
$$

Then, we have

Theorem 4.1. Let $F / \mathbb{F}_{q}$ be an algebraic function field defined over $\mathbb{F}_{q}$ of genus $g \geq 2$ and $h$ be its class number. For any set $D_{1} \subseteq \Delta_{1}$, any set $D_{2} \subseteq \Delta_{2}$, any finite set of integers $l=\left\{l_{r}\right\}_{r \in \Delta_{1}}$ and any finite set of integers $m=\left\{m_{r}\right\}_{r \in \Delta_{2}}$ such that

$$
l_{r} \geq 0, \quad \sum_{r \in \Delta_{1}} r l_{r} \leq g-1
$$

and

$$
m_{r} \geq 0, \quad \sum_{r \in \Delta_{2}} r m_{r} \leq g-2
$$


we have the following

$$
\frac{(q-1)^{2}\left(\Sigma_{1, \text { inf }}+\Sigma_{2, \text { inf }}\right)}{(g+1)(q+1)-B_{1}} \leq h \leq \frac{(q+1)^{2}\left(\Sigma_{1, \text { sup }}+\Sigma_{2, \text { sup }}\right)}{(g+1)(q+1)-B_{1}}
$$

where

$$
\begin{aligned}
& \Sigma_{1, \text { inf }}= \begin{cases}(a) & \prod_{r \in D_{1}}\left(\begin{array}{c}
B_{r}+l_{r} \\
l_{r}
\end{array}\right) \\
\text { or } & \left(\begin{array}{c}
B_{1}+g-1 \\
g-1
\end{array}\right)+ \\
(b) & \left.\left(\begin{array}{c}
B_{r}+\left\lfloor\frac{g-1}{r}\right\rfloor \\
\left\lfloor\frac{g-1}{r}\right\rfloor
\end{array}\right)-1\right)\left(\begin{array}{c}
B_{1}+((g-1) \bmod r) \\
(g-1) \bmod r
\end{array}\right) \\
\sum_{r \in D_{1}}\left(\begin{array}{c}
\text { with } B_{1} \geq 1,
\end{array}\right)\end{cases} \\
& \Sigma_{1, \text { sup }}=\prod_{r \in \Delta_{1}}\left(\begin{array}{c}
B_{r}+\left\lfloor\frac{g-1}{r}\right\rfloor \\
\left\lfloor\frac{g-1}{r}\right\rfloor
\end{array}\right),
\end{aligned}
$$

and

$$
\Sigma_{2, \text { inf }}= \begin{cases}(c) & q^{g-1} \prod_{r \in D_{2}} Q_{r, m_{r}} \\ o r & \\ (d) & q^{g-1}\left(Q_{1, g-2}+\sum_{r \in D_{2}} Q_{r,\left\lfloor\frac{g-2}{r}\right\rfloor}\right) \text { with } B_{1} \geq 1,\end{cases}
$$

and

$$
\Sigma_{2, \text { sup }}=q^{g-1} \prod_{r \in \Delta_{2}} Q_{r,\left\lfloor\frac{g-2}{r}\right\rfloor}
$$

where the quantity $Q_{r, s}$ is defined and estimated in Proposition 3.12.

Note that the formulas (a) and (c) can be used in all the cases and (b) and (d) only if $B_{1} \geq 1$. Note also that the quantities $\Sigma_{1, \text { inf }}$ and $\Sigma_{2, \text { inf }}$ can be chosen respectively among two bounds whose the quality depends on the studied situation.

The following theorem is a direct consequence of Theorem 4.1 and Proposition 3.12 (a).

Theorem 4.2. Let $F / \mathbb{F}_{q}$ be an algebraic function field defined over $\mathbb{F}_{q}$ of genus $g \geq 2$ and $h$ its class number. For any $r$ such that $1 \leq r \leq g-1$, let $B_{r}$ be the number of places of degree $r$ of $F / \mathbb{F}_{q}$.

For any set $D_{1}$, any set $D_{2}$, any finite set of integers $l=\left\{l_{r}\right\}_{r \in D_{1}}$ and any finite set of integers $m=\left\{m_{r}\right\}_{r \in D_{2}}$ such that

(1) $D_{1} \subseteq\{1, \cdots, g-1\}$;

(2) for any $r \in D_{1}$, we have $B_{r} \geq 1$;

(3) $D_{2} \subseteq\{1, \cdots, g-2\}$;

(4) for any $r \in D_{2}$, we have $B_{r} \geq 1$;

(5) $l_{r} \geq 0, \quad \sum_{r \in D_{1}} r l_{r} \leq g-1$;

(6) $m_{r} \geq 0, \quad \sum_{r \in D_{2}} r m_{r} \leq g-2$.

Then $h \geq h_{B R T}$ where

$$
\begin{array}{r}
h_{B R T}=\frac{(q-1)^{2}}{(g+1)(q+1)-B_{1}}\left(\prod_{r \in D_{1}}\left(\begin{array}{c}
B_{r}+l_{r} \\
l_{r}
\end{array}\right)\right. \\
\left.+q^{g-1} \prod_{r \in D_{2}}\left[\left(\frac{q^{r}}{q^{r}-1}\right)^{B_{r}}-B_{r}\left(\begin{array}{c}
B_{r}+m_{r} \\
B_{r}
\end{array}\right) \int_{0}^{\frac{1}{q^{r}}} \frac{\left(\frac{1}{q^{r}}-t\right)^{m_{r}}}{(1-t)^{B_{r}+m_{r}+1}} \mathrm{dt} .\right]\right) .
\end{array}
$$


Remark 4.3. Note that if we specialize to the case $r=1$ without knowing information about other degrees, the bound of Theorem 4.1 is exactly the same as the bound obtained in [5, Theorem 3.1] because of Remarks 3.5] and 3.14.

\section{LOWER BOUNDS ON THE CLASS NUMBER IN TOWERS}

In this section we study the class number of few recursive towers $\mathcal{F} / \mathbb{F}_{q}=$ $\left(F_{k} / \mathbb{F}_{q}\right)$ defined over different finite fields $\mathbb{F}_{q}$. In particular, we consider towers with $B_{r}\left(F_{k} / \mathbb{F}_{q}\right)>0$ for various $r \geq 1$. We study precisely the following parameters of each step $F_{k} / \mathbb{F}_{q}$ of those towers: the genus, the number of places of certain degrees, and the class number. Moreover, we study the possibly existence of at least a place of degree one. Let us first recall few definitions:

\section{Definition 5.1.}

(1) A tower over $\mathbb{F}_{q}$ is an infinite sequence $\mathcal{F} / \mathbb{F}_{q}=\left(F_{k} / \mathbb{F}_{q}\right)_{k \geq 0}$ of function fields $F_{k} / \mathbb{F}_{q}$ with the following properties:

(a) for all $k \geq 0$, the field $\mathbb{F}_{q}$ is algebraically closed in $F_{k}$ and each extension $F_{k+1} / F_{k}$ is finite separable such that $F_{k} \varsubsetneqq F_{k+1}$.

(b) The genera $g\left(F_{k}\right) \rightarrow \infty$ as $k \rightarrow \infty$.

(2) Let $\mathcal{F} / \mathbb{F}_{q}=\left(F_{k} / \mathbb{F}_{q}\right)_{k \geq 0}$ be a tower and $f(X, Y) \in \mathbb{F}_{q}[X, Y]$ be a nonconstant polynomial. Suppose that there exist elements $x_{k} \in F_{k}$ (for $k \geq 0$ ) such that

$$
F_{k+1}=F_{k}\left(x_{k+1}\right) \text { with } f\left(x_{k}, x_{k+1}\right)=0 \text { for all } n \geq 0 .
$$

Then we say that the tower $\mathcal{F} / \mathbb{F}_{q}$ is recursively defined by the polynomial $f(X, Y)$. In this case, we call that $\mathcal{F} / \mathbb{F}_{q}$ is a recursive tower.

(3) Let $\mathcal{F} / \mathbb{F}_{q}=\left(F_{k} / \mathbb{F}_{q}\right)_{k \geq 0}$ be a tower and $E$ be a finite separable extension of $F_{0}$. Suppose that $\mathcal{E} / \mathbb{F}_{q}=\left(E_{k} / \mathbb{F}_{q}\right)_{k \geq 0}$, with $E_{k}:=E F_{k}$ is a tower such that $E / F_{0}$ and $F_{k} / F_{0}$ are linearly disjoint for all $k \geq 0$. Then we call $\mathcal{E} / \mathbb{F}_{q}$ the composite tower of $\mathcal{F} / \mathbb{F}_{q}$ with $E$. When $\mathcal{F} / \mathbb{F}_{q}$ and $E$ are clear, we just say that $\mathcal{E} / \mathbb{F}_{q}$ is a composite tower.

(4) We will call a tower $\mathcal{F} / \mathbb{F}_{q}=\left(F_{k} / \mathbb{F}_{q}\right)_{k \geq 0}$ a quadratic tower if $\left[F_{k+1}: F_{k}\right]=2$ for all $k \geq 0$.

Remark 5.2. Let $\mathcal{F} / \mathbb{F}_{q}=\left(F_{k} / \mathbb{F}_{q}\right)_{k \geq 0}$ be a recursive tower defined by the polynomial $f(X, Y) \in \mathbb{F}_{q}[X, Y]$ and $\mathcal{E} / \mathbb{F}_{q}$ be a composite tower of $\mathcal{F} / \mathbb{F}_{q}$ with an extension $E$ of $F_{0}$. Then $\mathcal{E} / \mathbb{F}_{q}$ is a recursive tower defined by the same polynomial $f(X, Y)$.

Let $\mathbb{P}(F)$ be the set of places of $F / \mathbb{F}_{q}$ and let $v_{P}$ be the discrete valuation associated to the place $P \in \mathbb{P}(F)$. Let $E / F$ be a finite separable extension of the function field $F$ over $\mathbb{F}_{q}, P \in \mathbb{P}(F), Q \in \mathbb{P}(E)$ be a place lying above $P$. Then we write $Q \mid P$ and denote $e(Q \mid P)$ the ramification index of $Q \mid P, f(Q \mid P)$ the relative degree of $Q \mid P, d(Q \mid P)$ the different exponent of $Q \mid P$ and $\operatorname{Diff}(E / F)$ the different of $E / F$. We denote by $(x=a)$ the place of the rational function field $\mathbb{F}_{q}(x)$ which is a zero of $x-a$ for $a \in \mathbb{F}_{q}$, or the pole of $x$ for $a=\infty$.

Definition 5.3. Let $\mathcal{F}=\left(F_{n}\right)_{n \geq 0}$ be a tower over $\mathbb{F}_{q}$. Then

$$
R(\mathcal{F}):=\left\{P \in \mathbb{P}\left(F_{0}\right) \mid P \text { is ramified in } F_{k} \text { for some } k \geq 0\right\}
$$

is called the ramification locus of $\mathcal{F}$. The set

$$
\operatorname{Split}(\mathcal{F}):=\left\{P \in \mathbb{P}\left(F_{0}\right) \mid P \text { splits completely in } F_{k} \text { for all } k \geq 1\right\}
$$

is called the splitting locus of $\mathcal{F}$. 
For a tower $\mathcal{F} / \mathbb{F}_{q}$, let $N=N\left(\mathcal{F} / \mathbb{F}_{q}\right)$ be a set of integers $r \geq 1$ such that $\beta_{r}\left(\mathcal{F} / \mathbb{F}_{q}\right)>0$.

5.1. Case $N=\{r\}$. In this section we give some examples of towers having only one positive invariant $\beta_{r}$ or having only one known positive invariant $\beta_{r}$ for any $r \geq 1$. These examples are obtained from asymptotically good towers with respect to the rational places over $\mathbb{F}_{q^{r}}$, i.e., with $\beta_{1}>0$ or from the descent of such towers on the ground field $\mathbb{F}_{q}$.

5.1.1. Example 1. This example concerns the first tower of Garcia-Stichtenoth 8 reaching the Drinfeld-Vladut bound over $\mathbb{F}_{q^{r}}$ where $q^{r}$ is a square. This tower is here denoted by $\mathcal{H} / \mathbb{F}_{q^{r}}$. We study bounds of the class number of all the steps of this tower as well as those of its descent tower $\mathcal{F} / \mathbb{F}_{q}$ to $\mathbb{F}_{q}$, which reaches the DrinfeldVladut bound of order $r$. Note that in these two cases, we know the exact value of the genus of each step. But in the case of the tower $\mathcal{H} / \mathbb{F}_{q^{r}}$, it is interesting to see that we obtain estimations of class number from the value (or from lower bound) of the number of places of degree one. However, in the case of the tower $\mathcal{F} / \mathbb{F}_{q}$, we can obtain estimations of class numbers from only the value (or from lower bound) of the quantity $\sum_{i \mid r} i B_{i}\left(F_{k}\right)$. These two towers illustrate two different situations where one can estimate class numbers.

Proposition 5.4. Let $q$ be a prime power and $r \geq 1$ be an integer such that $q^{r}$ is a square. Consider the tower $\mathcal{H} / \mathbb{F}_{q^{r}}=\left(H_{k} / \mathbb{F}_{q^{r}}\right)_{k>0}$ defined recursively by the polynomial

$$
f(X, Y)=Y^{q^{r / 2}} X^{q^{r / 2}-1}+Y-X^{q^{r / 2}} \in \mathbb{F}_{q}[X, Y]
$$

and the rational function field $F_{0}=\mathbb{F}_{q}\left(x_{0}\right)$. Then the tower $\mathcal{H} / \mathbb{F}_{q^{r}}$ and its descent $\mathcal{F} / \mathbb{F}_{q}=\left(F_{k} / \mathbb{F}_{q}\right)_{k \geq 0}$ which is such that $\mathcal{H} / \mathbb{F}_{q^{r}}=\mathcal{F} / \mathbb{F}_{q} \otimes_{\mathbb{F}_{q}} \mathbb{F}_{q^{r}}$ verify the following properties:

(i) $B_{1}\left(F_{k}\right) \geq 1$;

(ii) a) if $q \equiv 1 \bmod 2$ then for all $k \geq 2$,

$$
\sum_{i \mid r} i B_{i}\left(F_{k}\right)=\left(q^{r}-1\right) q^{r k / 2}+2 q^{r / 2}=B_{1}\left(H_{k}\right) .
$$

b) if $q \equiv 0 \bmod 2$ then

$$
\begin{gathered}
\sum_{i \mid r} i B_{i}\left(F_{2}\right)=\left(q^{r}-1\right)\left(q^{r}\right)+2 q^{r / 2}=B_{1}\left(H_{2}\right), \\
\sum_{i \mid r} i B_{i}\left(F_{3}\right)=\left(q^{r}-1\right) q^{3 r / 2}+q^{r}+q^{r / 2}=B_{1}\left(H_{3}\right), \\
\sum_{i \mid r} i B_{i}\left(F_{k}\right)=\left(q^{r}-1\right) q^{r k / 2}+2 q^{r}=B_{1}\left(H_{k}\right) \text { for all } k \geq 4 .
\end{gathered}
$$

(iii) The genus $g\left(H_{k}\right)=g\left(F_{k}\right)$ and

$$
g\left(F_{k}\right)= \begin{cases}\left(q^{r / 2}+1\right) q^{r k / 2}-\left(q^{r / 2}+2\right) q^{r k / 4}+1 & \text { if } k \text { even } \\ \left(q^{r / 2}+1\right) q^{r k / 2}-\frac{1}{2}\left(q^{r}+3 q^{r / 2}+1\right) q^{(r k-r) / 4}+1 & \text { if } k \text { odd } .\end{cases}
$$

(iv) $\beta_{r}\left(\mathcal{F} / \mathbb{F}_{q}\right)=\frac{q^{r / 2}-1}{r}$. 
Proof. The tower $\mathcal{H} / \mathbb{F}_{q^{r}}=\left(H_{k} / \mathbb{F}_{q^{r}}\right)_{k \geq 0}$ is the well-known Garcia-Stichtenoth tower over $\mathbb{F}_{q^{r}}$ studied in [8]. Since $\mathcal{H} / \mathbb{F}_{q^{r}}$ is the constant field extension of $\mathcal{F} / \mathbb{F}_{q}$, the assertions (ii) and (iii) are respectively straightforward consequences of Remark 3.4 and Theorem 2.10 in [8]. Assertion (iv) follows from assertions (ii) and (iii). To prove (i), we consider the pole $P_{\infty}$ of $x_{0}$ in $H_{0} / \mathbb{F}_{q^{r}}$. By [8, Lemma 2.1], the place $P_{\infty}$ is totally ramified in $H_{k} / H_{0}$ for all $k \geq 0$. Since $P_{\infty}$ is invariant under the action of the Galois group $\operatorname{Gal}\left(\mathbb{F}_{q^{r}} / \mathbb{F}_{q}\right)$, its restriction $P_{\infty} \cap F_{0}$ in $F_{0} / \mathbb{F}_{q}$ is then totally ramified in $F_{k} / \mathbb{F}_{q}$, and so $(i)$ follows.

Let us recall the following fact:

Remark 5.5. Let $\mathcal{F} / \mathbb{F}_{q}=\left(F_{k} / \mathbb{F}_{q}\right)_{k \geq 0}$ be a sequence with $g\left(F_{k}\right) \rightarrow \infty$ as $k \rightarrow \infty$. Consider the constant field extension $\mathcal{F} / \mathbb{F}_{q^{r}}=\left(F_{k} \mathbb{F}_{q^{r}} / \mathbb{F}_{q^{r}}\right)_{k \geq 0}$ of $\mathcal{F} / \mathbb{F}_{q}$, for some $r \geq 1$. It is known from [5] that

$$
\beta_{r}\left(\mathcal{F} / \mathbb{F}_{q}\right)=\frac{q^{r / 2}-1}{r} \text { if and only if } \beta_{1}\left(\mathcal{F} / \mathbb{F}_{q^{r}}\right)=q^{r / 2}-1 .
$$

Now, let us give an example concerning the estimation of the class number in all the steps of the towers $\mathcal{H} / \mathbb{F}_{q^{r}}=\left(H_{k} / \mathbb{F}_{q^{r}}\right)_{k \geq 0}$ and $\mathcal{F} / \mathbb{F}_{q}=\left(F_{k} / \mathbb{F}_{q}\right)_{k \geq 0}$ in the case of $q=2$ and $r=2$.

Proposition 5.6. The steps of the towers $\mathcal{H} / \mathbb{F}_{q^{2}}=\left(H_{k} / \mathbb{F}_{q^{2}}\right)_{k \geq 0}$ and $\mathcal{F} / \mathbb{F}_{q}=$ $\left(F_{k} / \mathbb{F}_{q}\right)_{k \geq 0}$ defined in Proposition 5.4 with $q=2$ and $r=2$ have class number such that

$$
h\left(H_{k} / \mathbb{F}_{q^{2}}\right) \geq h_{B R T 1, k}
$$

and

$$
h\left(F_{k} / \mathbb{F}_{q}\right) \geq h_{B R T 2, k}
$$

where

$$
\begin{array}{r}
h_{B R T 1, k}=\frac{\left(q^{r}-1\right)^{2}}{\left(g_{k}+1\right)\left(q^{r}+1\right)-B_{1}\left(H_{k}\right)}\left(\left(\begin{array}{c}
B_{1}\left(H_{k}\right)+g_{k}-1 \\
B_{1}\left(H_{k}\right)
\end{array}\right)\right. \\
+q^{r\left(g_{k}-1\right)}\left[\left(\frac{q^{r}}{q^{r}-1}\right)^{B_{1}\left(H_{k}\right)}-\right. \\
\left.\left.B_{1}\left(H_{k}\right)\left(\begin{array}{c}
B_{1}\left(H_{k}\right)+g_{k}-2 \\
B_{1}\left(H_{k}\right)
\end{array}\right) \int_{0}^{\frac{1}{q^{r}}} \frac{\left(\frac{1}{q^{r}}-t\right)^{g_{k}-2}}{(1-t)^{B_{1}\left(H_{k}\right)+g_{k}-1} \mathrm{dt} .}\right]\right)
\end{array}
$$

and

$$
\begin{array}{r}
h_{B R T 2, k}=\frac{(q-1)^{2}}{\left(g_{k}+1\right)(q+1)-B_{1}^{\prime}\left(F_{k}\right)}\left(\prod_{i=1}^{2}\left(\begin{array}{c}
B_{i}^{\prime}\left(F_{k}\right)+m_{i} \\
B_{i}^{\prime}\left(F_{k}\right)
\end{array}\right)\right. \\
+q^{g_{k}-1} \prod_{i=1}^{2}\left[\left(\frac{q^{i}}{q^{i}-1}\right)^{B_{i}^{\prime}\left(F_{k}\right)}-\right. \\
B_{i}^{\prime}\left(F_{k}\right)\left(\begin{array}{c}
B_{i}^{\prime}\left(F_{k}\right)+m_{i} \\
B_{i}^{\prime}\left(F_{k}\right)
\end{array}\right) \int_{0}^{\frac{1}{q^{i}}} \frac{\left(\frac{1}{q^{i}}-t\right)^{m_{i}}}{\left.\left.(1-t)^{B_{i}^{\prime}\left(F_{k}\right)+m_{i}+1} \mathrm{dt} .\right]\right)}
\end{array}
$$

with $B_{1}^{\prime}\left(F_{k}\right)=2, B_{2}^{\prime}\left(F_{k}\right)=\left\lfloor\frac{B_{1}\left(H_{k}\right)-2}{2}\right\rfloor$ and $\sum_{i=1}^{2} i m_{i} \leq g_{k}-2$ for all $k \geq 2$. 
Proof. The bound $h_{B R T 1, k}$ follows directly from Theorem 4.2 By Section 2 it is clear that the lower bounds of the class number $h$ are increasing with the number of effective divisors constructed with places of degree less or equal to a fixed degree. Here, we do not know $B_{1}\left(F_{k}\right)$ nor $B_{2}\left(F_{k}\right)$ but we know the value of the quantity $B_{1}\left(F_{k}\right)+2 B_{2}\left(F_{k}\right)$. This quantity is even then $B_{1}\left(F_{k}\right)$ is even. Hence, if we replace $B_{1}\left(F_{k}\right) \geq 2$ by $B_{1}^{\prime}\left(F_{k}\right)=2$ and $B_{2}\left(F_{k}\right)$ by $B_{2}^{\prime}\left(F_{k}\right)=\left\lfloor\frac{\left(B_{1}\left(F_{k}\right)+2 B_{2}\left(F_{k}\right)\right)-2}{2}\right\rfloor$, we obtain a lower bound on the number of effective divisors and then a lower bound for $h_{B R T 2, k}$.

\section{Numerical estimations:}

Here are some effective numerical estimations of the class numbers of some steps of the towers $\mathcal{H} / \mathbb{F}_{4}$ and $\mathcal{F} / \mathbb{F}_{2}$ given in Proposition [5.6.

More precisely, the following table gives estimations of the class number of the first steps of the original tower $\mathcal{H}$ of Garcia-Stichtenoth reaching the DrinfeldVladut bound. We make these estimations with $q=4$ and we only use the places of degree one. Moreover, recall that we denote by $h_{A H L}$ the adapted bound in this context extracted from Theorem 1.5 (namely bound (III)) obtained by Aubry, Halaoui, and Lachaud. In particular, we see that our bound $h_{B R T}$ of Proposition 5.6 is better than $H_{A H L}$ for $k \geq 3$. Note also that in this context the bound $h_{B R}$ obtained in [5, Theorem 3.1] gives the same estimations because in the particular case $r=1$, these bounds are the same as those given by $h_{B R T}$ (cf. Remark 4.3).

\begin{tabular}{|l|c|c|c|c|c|c|}
\hline$q^{2}$ & step k & $g_{k}$ & $B_{1}\left(H_{k} / \mathbb{F}_{q}\right)$ & $m_{1}$ & $h_{B R T 1, k}=h_{B R}$ & $h_{A H L}$ \\
\hline 4 & 2 & 5 & 16 & 3 & 7434 & 12240 \\
\hline 4 & 3 & 14 & 30 & 12 & 64916794126 & 46784340680 \\
\hline 4 & 4 & 33 & 56 & 31 & $1.43 \times 10^{25}$ & $0.075 \times 10^{25}$ \\
\hline
\end{tabular}

Now, in the following table, we give estimations of the class number of the first steps of the descent tower of Garcia-Stichtenoth reaching the Drinfeld-Vladut bound of order $r=2$. We make these estimations with $q=2$ and we only use the places of degree one and two. Note that for any $k$, Condition $B_{1}\left(H_{k} / \mathbb{F}_{q}\right) \geq g_{k}\left(q^{1 / 2}-1\right)$ of Theorem 2.4 in [1] (or [2]) is not satisfied. Hence, we must not use Bound (III) of Theorem 1.5. Moreover, recall that it is clear that Bound (II) gives no significant result because of the factor $q^{g}$ in the denominator.

\begin{tabular}{|c|c|c|c|c|c|c|c|}
\hline $\mathrm{q}$ & step k & $g_{k}$ & $B_{1}^{\prime}\left(F_{k} / \mathbb{F}_{q}\right)$ & $B_{2}^{\prime}\left(F_{k} / \mathbb{F}_{q}\right)$ & $m_{1}$ & $m_{2}$ & $h_{B R T 2, k}$ \\
\hline 2 & 2 & 5 & 2 & 7 & 1 & 1 & 7 \\
\hline 2 & 3 & 14 & 2 & 14 & 2 & 5 & 21257 \\
\hline 2 & 4 & 33 & 2 & 27 & 5 & 13 & 343733443618 \\
\hline
\end{tabular}

Remark 5.7. Recall that for $H_{k} / \mathbb{F}_{4}$,

$$
\Sigma_{1, k, \text { inf }}=\left(\begin{array}{c}
B_{1}\left(H_{k}\right)+g_{k}-1 \\
B_{1}\left(H_{k}\right)
\end{array}\right)
$$


and

$$
\begin{array}{r}
\Sigma_{2, k, \text { inf }}=q^{g_{k}-1}\left[\left(\frac{q^{r}}{q^{r}-1}\right)^{B_{1}\left(H_{k}\right)}-\right. \\
B_{1}\left(H_{k}\right)\left(\begin{array}{c}
B_{1}\left(H_{k}\right)+g_{k}-2 \\
B_{1}\left(H_{k}\right)
\end{array}\right) \int_{0}^{\frac{1}{q^{r}}} \frac{\left(\frac{1}{q^{r}}-t\right)^{g_{k}-2}}{\left.(1-t)^{B_{1}\left(H_{k}\right)+g_{k}-1} \mathrm{dt}\right] .}
\end{array}
$$

By our numerical simulations in the tower $\mathcal{H} / \mathbb{F}_{q^{r}}$, we have $\frac{\Sigma_{1, k, \text { inf }}}{\Sigma_{2, k, \text { inf }}} \leq 0.0054$ for all $k \geq 4$ and in fact, this ratio tends to zero when $k$ tends to the infinity. Moreover, let us set

$$
C_{k}=\left(\frac{q^{r}}{q^{r}-1}\right)^{B_{1}\left(H_{k}\right)}
$$

and

$$
D_{k}=B_{1}\left(H_{k}\right)\left(\begin{array}{c}
B_{1}\left(H_{k}\right)+g_{k}-2 \\
B_{1}\left(H_{k}\right)
\end{array}\right) \int_{0}^{\frac{1}{q^{r}}} \frac{\left(\frac{1}{q^{r}}-t\right)^{g_{k}-2}}{(1-t)^{B_{1}\left(H_{k}\right)+g_{k}-1}} \mathrm{dt} .
$$

Then the ratio $\frac{D_{k}}{C_{k}} \leq 0.01$ for all $k \geq 4$. In fact, this ratio tends to zero when $k$ tends to the infinity. The situation in the tower $\mathcal{F} / \mathbb{F}_{q}$ is similar. Moreover, in the numerical estimations concerning the tower $\mathcal{F} / \mathbb{F}_{q}$, we have specialized $l_{i}=m_{i}$ for $i=1,2$ from the general formula in Theorem 4.1. Indeed, the realized numerical simulations on few steps give no improvement of the bounds for the class number of $F_{k}$ by distinguishing $l_{i}$ and $m_{i}$ in the quantities

$$
\Sigma_{1, k}=\prod_{i=1}^{2}\left(\begin{array}{c}
B_{i}\left(F_{k}\right)+l_{i} \\
B_{i}\left(F_{k}\right)
\end{array}\right)
$$

and

$$
\Sigma_{2, k}=\prod_{i=1}^{2}\left[\left(\frac{q^{i}}{q^{i}-1}\right)^{B_{i}^{\prime}\left(F_{k}\right)}-B_{i}^{\prime}\left(\begin{array}{c}
B_{i}^{\prime}\left(F_{k}\right)+m_{i} \\
B_{i}^{\prime}\left(F_{k}\right)
\end{array}\right) \int_{0}^{\frac{1}{q^{i}}} \frac{\left(\frac{1}{q^{i}}-t\right)^{m_{i}}}{(1-t)^{B_{i}^{\prime}\left(F_{k}\right)+m_{i}+1}} \mathrm{dt}\right] .
$$

5.1.2. Example 2. This example concerns a tower defined over cubic finite fields constructed by Bassa, Garcia and Stichtenoth [6]. We study bounds of the class number of all the steps of this tower as well as those of its descent tower over $\mathbb{F}_{q}$. Note that in these two cases, we only know lower and upper bounds for the genus of each step. Moreover, even if we do not know whether there exist rational places in each step of the descent tower over $\mathbb{F}_{q}$, we can give good estimations for the class number of all the steps of this tower.

Proposition 5.8. Let $q$ be a prime power and $\mathcal{H} / \mathbb{F}_{q^{3}}=\left(H_{k} / \mathbb{F}_{q^{3}}\right)_{k \geq 0}$ the tower defined recursively by the polynomial

$$
f(X, Y)=\left(Y^{q}-Y\right)^{q-1}+1+\frac{X^{q(q-1)}}{\left(X^{q-1}-1\right)^{q-1}} \in \mathbb{F}_{q}[X, Y] .
$$

The tower $\mathcal{H} / \mathbb{F}_{q^{3}}$ and the descent tower $\mathcal{F} / \mathbb{F}_{q}=\left(F_{k} / \mathbb{F}_{q}\right)_{k \geq 0}$ defined by its constant field extension $\mathcal{H} / \mathbb{F}_{q^{3}}=\mathcal{F} / \mathbb{F}_{q} \otimes_{\mathbb{F}_{q}} \mathbb{F}_{q^{3}}$ have the following properties:

(i) $B_{1}\left(H_{k}\right) \geq 3 B_{3}\left(F_{k}\right)$ with $B_{3}\left(F_{k}\right) \geq \frac{\left(q^{3}-q^{2}-q+1\right) q^{k+1}}{3}$ for all $k \geq 1$. 
(ii) for all $k \geq 2$, one has that

$$
\frac{1}{2}\left(q^{4}+q^{3}-6 q^{2}+4 q\right) q^{k-1}-q^{3}+q^{2}+1 \leq g\left(F_{k}\right) \leq \frac{\left(q^{2}+q-2\right) q^{k+1}}{2} .
$$

For $k=1$, the first equality holds.

(iii) $\beta_{3}\left(\mathcal{F} / \mathbb{F}_{q}\right) \geq \frac{2\left(q^{2}-1\right)}{3(q+2)}$.

Proof. Let $H_{0}=\mathbb{F}_{q^{3}}\left(x_{0}\right)$ be the rational function field and $H_{1}=\mathbb{F}_{q^{3}}\left(x_{0}, x_{1}\right)$ where $f\left(x_{0}, x_{1}\right)=0$. We know the following from [6, Theorems 2.2, 3.4 and 6.5]:

(a) For all $k \geq 0$, the extensions $H_{k+1} / H_{k}$ are Galois with $\left[H_{1}: H_{0}\right]=q(q-1)$ and $\left[H_{k+1}: H_{k}\right]=q$ for all $k \geq 1$.

(b) The descent sequence of $\mathcal{H} / \mathbb{F}_{q^{3}}$ to any field $K$ containing $\mathbb{F}_{q}$ is a tower.

(c) The genus $g\left(H_{k}\right) \leq \frac{\left(q^{3}+q^{2}-2 q\right) q^{k}}{2}$ for all $k \geq 0$.

(d) $\operatorname{Split}(\mathcal{H}) \supseteq\left\{\left(x_{0}=\alpha\right) \mid \alpha \in \mathbb{F}_{q^{3}} \backslash \mathbb{F}_{q}\right\}$.

(i) Since

$$
B_{1}\left(H_{0}\right)=B_{1}\left(F_{0}\right)+3 B_{3}\left(F_{0}\right),
$$

we have that $B_{3}\left(F_{0}\right)=\frac{q^{3}-q}{3}$. Obviously, any degree 3 place $P \in \mathbb{P}\left(F_{0}\right)$ splits completely in $H_{0}$ and its extensions are in the set $\operatorname{Split}(\mathcal{H})$. Hence, clearly, $P$ splits completely in $F_{k}$ for all $k \geq 0$. Thus, for all $k \geq 0$, we obtain that

$$
B_{3}\left(F_{k}\right) \geq B_{3}\left(F_{0}\right)\left[F_{k}: F_{0}\right],
$$

and hence (i) follows.

(ii) We first note that after taking the constant field extension the genera do not change, see [14, Theorem 3.6.3(b)]. The second inequality follows from (b) as $g\left(F_{k}\right)=g\left(H_{k}\right)$. To prove the first inequality, we consider the sequence $\mathcal{F}^{\prime} / \mathbb{F}_{q^{2}}=$ $\left(F_{k}^{\prime} / \mathbb{F}_{q^{2}}\right)_{k \geq 0}$, where $F_{k}^{\prime}=F_{k} \mathbb{F}_{q^{2}}$. Then $g\left(F_{k}\right)=g\left(F_{k}^{\prime}\right)$ for all $k \geq 0$. We first compute $g\left(F_{1}^{\prime}\right)$. Let $Q$ be a place of $F_{1}^{\prime}$ and $P=Q \cap F_{0}^{\prime}$. By using [6, Lemma 5.2] and [14, Definition 7.4.12 and Theorem 3.5.1], we obtain that

$$
d(Q \mid P)=\left\{\begin{array}{l}
0 \text { if } P=\left(x_{0}=0\right) \\
2 q-2 \text { if } P=\left(x_{0}=\infty\right) \text { or }\left(x_{0}=\alpha\right) \text { with } \alpha \in \mathbb{F}_{q}^{*} \\
q-2 \text { if } P=\left(x_{0}=\beta\right) \text { with } \beta \in \mathbb{F}_{q^{2}} \backslash \mathbb{F}_{q} .
\end{array}\right.
$$

Let $s=\#\left\{Q \in \mathbb{P}\left(F_{1}^{\prime}\right): Q \mid P\right\}$ and $f=f(Q \mid P)$ (note that $F_{1}^{\prime} / F_{0}^{\prime}$ is Galois). By the same lemma,

$$
s f=\left\{\begin{array}{l}
q-1 \text { if } P=\left(x_{0}=\infty\right) \text { or }\left(x_{0}=\alpha\right) \text { with } \alpha \in \mathbb{F}_{q}^{*} \\
q \text { if } P=\left(x_{0}=\beta\right) \text { with } \beta \in \mathbb{F}_{q^{2}} \backslash \mathbb{F}_{q} .
\end{array}\right.
$$

Hence,

$$
\begin{aligned}
\operatorname{deg} \operatorname{Diff}\left(F_{1}^{\prime} / F_{0}^{\prime}\right) & =\sum_{P} \sum_{Q} d(Q \mid P) \operatorname{deg} Q \\
& =(2 q-2)(q-1)+(2 q-2)(q-1)^{2}+q(q-2)\left(q^{2}-q\right) \\
& =q^{4}-q^{3}-2 q^{2}+2 q .
\end{aligned}
$$

The Hurwitz Genus Formula [14] for the extension $F_{1}^{\prime} / F_{0}^{\prime}$ now yields that

$$
g\left(F_{1}^{\prime}\right)=\frac{1}{2}\left(q^{4}-q^{3}-4 q^{2}+4 q+2\right) .
$$


Next, we estimate $g\left(F_{k}^{\prime}\right)$ for any $k \geq 2$. Let $Q_{k}$ be a place of $F_{k}^{\prime}$ and $Q=Q_{k} \cap F_{1}^{\prime}$. Suppose that $Q$ lies above one of the following places:

- $\left(x_{0}=\infty\right)$ or $\left(x_{0}=\alpha\right)$ with $\alpha \in \mathbb{F}_{q}^{*}$,

- $\left(x_{0}=\beta\right)$ with $\beta \in \mathbb{F}_{q^{2}} \backslash \mathbb{F}_{q}$ and $\left(x_{1}=\alpha\right)$ with $\alpha \in \mathbb{F}_{q}^{*}$.

By [6, Lemmas 5.2, 6.3, 6.4] (also c.f.proof of [6, Theorem 6.5]), we have that

$$
e\left(Q_{k} \mid Q\right)=q^{k-1} \quad \text { and } \quad d\left(Q_{k} \mid Q\right)=2\left(e\left(Q_{k} \mid Q\right)-1\right)=2\left(q^{k-1}-1\right) .
$$

Hence, using (29) and (31) yields that

$$
\begin{aligned}
\operatorname{deg} \operatorname{Diff}\left(F_{k}^{\prime} / F_{1}^{\prime}\right) & \geq \sum_{Q} \sum_{Q_{k}} d\left(Q_{k} \mid Q\right) \operatorname{deg} Q_{k} \\
& =2\left(q^{k-1}-1\right)\left[(q-1)+(q-1)^{2}+\left(q^{2}-q\right)(q-1)\right] \\
& =2\left(q^{3}-q^{2}\right)\left(q^{k-1}-1\right) .
\end{aligned}
$$

Then using (30) and the Hurwitz Genus Formula [14 for the extension $F_{k}^{\prime} / F_{1}^{\prime}$ gives that

$$
g\left(F_{k}\right)=g\left(F_{k}^{\prime}\right) \geq \frac{1}{2}\left(q^{4}+q^{3}-6 q^{2}+4 q\right) q^{k-1}-q^{3}+q^{2}+1 .
$$

Note that when $k=1$, the equality in (32) holds.

(iii) follows from (i) and (ii).

Proposition 5.9. The steps of the towers $\mathcal{H} / \mathbb{F}_{q^{3}}=\left(H_{k} / \mathbb{F}_{q^{3}}\right)_{k \geq 0}$ and $\mathcal{F} / \mathbb{F}_{q}=$ $\left(F_{k} / \mathbb{F}_{q}\right)_{k \geq 0}$ defined in Proposition 5.8 have a class number such that

$$
h\left(H_{k} / \mathbb{F}_{q^{3}}\right) \geq h_{B R T 1, k}
$$

and

where

$$
h\left(F_{k} / \mathbb{F}_{q}\right) \geq h_{B R T 2, k}
$$

$$
\begin{array}{r}
h_{B R T 1, k}=\frac{\left(q^{3}-1\right)^{2}}{\left(g_{k}+1\right)\left(q^{3}+1\right)-B_{1, k}}\left(\left(\begin{array}{c}
B_{1, k}+g_{k}-1 \\
B_{1, k}
\end{array}\right)\right. \\
+q^{3\left(g_{k}-1\right)}\left[\left(\frac{q^{3}}{q^{3}-1}\right)^{B_{1, k}}-\right. \\
\left.\left.B_{1, k}\left(\begin{array}{c}
B_{1, k}+g_{k}-2 \\
B_{1, k}
\end{array}\right) \int_{0}^{\frac{1}{q^{3}}} \frac{\left(\frac{1}{q^{r}}-t\right)^{g_{k}-2}}{(1-t)^{B_{1, k}+g_{k}-1}} \mathrm{dt} .\right]\right)
\end{array}
$$

and

$$
\begin{array}{r}
h_{B R T 2, k}=\frac{(q-1)^{2}}{\left(g_{k}+1\right)(q+1)}\left(\left(\begin{array}{c}
B_{3, k}+\left\lfloor\frac{g_{k}-1}{3}\right\rfloor \\
B_{3, k}
\end{array}\right)\right. \\
+q^{g_{k}-1}\left[\left(\frac{q^{3}}{q^{3}-1}\right)^{B_{3, k}}-\right. \\
\left.\left.B_{3, k}\left(\begin{array}{c}
B_{3, k}+\left\lfloor\frac{g_{k}-2}{3}\right\rfloor \\
B_{3, k}
\end{array}\right) \int_{0}^{\frac{1}{q^{3}}} \frac{\left(\frac{1}{q^{3}}-t\right)^{\left\lfloor\frac{g_{k}-2}{3}\right\rfloor}}{(1-t)^{B_{3, k}+\left\lfloor\frac{g_{k}-2}{3}\right\rfloor+1}} \mathrm{dt} .\right]\right)
\end{array}
$$

with $B_{3, k}=\frac{\left(q^{3}-q^{2}-q+1\right) q^{k+1}}{3}, B_{1, k}=\left(q^{3}-q^{2}-q+1\right) q^{k+1}$ and $g_{k}=\frac{1}{2}\left(q^{4}+q^{3}-\right.$ $\left.6 q^{2}+4 q\right) q^{k-1}-q^{3}+q^{2}+1$ for all $k \geq 1$ 
Proof. The bound $h_{B R T 1, k}$ follows directly from Theorem 4.2 by using the lower bound on the genus given by Proposition 5.8 since the functions $h_{B R T 1, k}$ and $h_{B R T 2, k}$ with respect to the genus are increasing. Moreover, we apply Theorem 4.2 by using the lower bound on the number of places of degree one given by Proposition 5.8 with the parameters $l_{1}=g_{k}-1$ and $m_{1}=g_{k}-2$. The bound $h_{B R T 2, k}$ follows directly from Theorem 4.2 as in the previous case by using the lower bound on the number of places of degree 3 given by Proposition 5.8 with the parameters $l_{3}=\left\lfloor\frac{g_{k}-1}{3}\right\rfloor$ and $m_{3}=\left\lfloor\frac{g_{k}-2}{3}\right\rfloor$.

\section{Numerical estimations:}

Here are some effective numerical estimations of the class numbers of some steps of the towers $\mathcal{H} / \mathbb{F}_{q^{3}}$ and $\mathcal{F} / \mathbb{F}_{q}$ given by Proposition $[5.9$ with $q=2$.

\begin{tabular}{|l|c|c|c|c|c|r|}
\hline$q^{3}$ & step k & $g_{k}$ & $B_{1, k}$ & $l_{1}$ & $m_{1}$ & $h_{B R T 1, k}$ \\
\hline 8 & 2 & 5 & 24 & 4 & 3 & 125537 \\
\hline 8 & 3 & 13 & 48 & 12 & 11 & $2.556 \times 10^{13}$ \\
\hline 8 & 4 & 29 & 96 & 28 & 27 & $2.010 \times 10^{30}$ \\
\hline
\end{tabular}

\begin{tabular}{|l|c|c|c|c|c|r|}
\hline $\mathrm{q}$ & step k & $g_{k}$ & $B_{3, k}$ & $l_{3}$ & $m_{3}$ & $h_{B R T 2, k}$ \\
\hline 2 & 2 & 5 & 8 & 1 & 1 & 3 \\
\hline 2 & 3 & 13 & 16 & 4 & 3 & 771 \\
\hline 2 & 4 & 29 & 32 & 9 & 9 & 212127395 \\
\hline
\end{tabular}

5.2. General case $N=\left\{r_{i}\right\}_{i}$. In this section, we first recall a method for constructing towers of function fields $\mathcal{F} / \mathbb{F}_{q}$ with various invariants $\beta_{r}(\mathcal{F})$ being positive. This method is based upon the compositum of asymptotically good towers with different suitable extensions. Then we give some examples of explicit towers having several positive invariants $\beta_{r_{i}}$, for which we describe precisely several parameters (such as genus, rational places, etc.). Then we give an estimation of class number of each step of these towers.

Let us recall a useful lemma concerning the genera in towers (See [10, Theorem 3.6]) which we will use often in the subsequent sections.

Lemma 5.10. Let $\mathcal{F} / \mathbb{F}_{q}=\left(F_{k} / \mathbb{F}_{q}\right)_{k \geq 0}$ be a tower and $E / F_{0}$ be a finite separable extension such that $R(\mathcal{F})$ is finite and all $P \in R(\mathcal{F})$ are tame in $E$. Suppose that $\mathcal{E} / \mathbb{F}_{q}=\left(E_{k} / \mathbb{F}_{q}\right)_{k \geq 0}$, with $E_{k}=E F_{k}$, is a composite tower. Set $m=[E: F]$. Then for all $k \geq 0$,

$$
g\left(E_{k}\right)=\left(g(E)-m g(F)+m-\frac{s+2}{2}\right)\left[E_{k}: E\right]+m\left(g\left(F_{k}\right)-1\right)+\frac{r(k)}{2}+1,
$$

where

$$
\begin{aligned}
s & =\sum_{\substack{Q \in \mathbb{P}(E) \\
Q \cap F \in R(\mathcal{F})}} d(Q \mid Q \cap F) \operatorname{deg} Q \quad \text { and } \\
r(k) & =\sum_{P \in R(\mathcal{F})} \sum_{\substack{P_{k} \in \mathbb{P}\left(F_{k}\right) \\
P_{k} \mid P}} \operatorname{deg} P_{k} \sum_{\substack{Q_{k} \in \mathbb{P}\left(E_{k}\right) \\
Q_{k} \mid P_{k}}}\left(e\left(Q_{k} \mid P_{k}\right)-1\right) f\left(Q_{k} \mid P_{k}\right) .
\end{aligned}
$$


5.2.1. Family 1. We begin with an example given in [7].

Proposition 5.11. Let $N \subset \mathbb{N}$ be a finite set, $m=\sum_{i \in N} i$, and $q$ be a prime power with $(m, q)=1$. Consider the tower $\mathcal{F} / \mathbb{F}_{q^{2}}$ given in Proposition 5.4. Let $E=F_{0}(z)$ with $z$ a root of the polynomial

$$
\varphi(T)=\prod_{i \in N} g_{i}(T)-x_{0}^{q^{2}}+x_{0} \in F_{0}[T],
$$

where $g_{i}(T) \in \mathbb{F}_{q^{2}}[T]$ is a monic, irreducible polynomial of $\operatorname{deg} g_{i}(T)=i$, for each $i \in N$. Then the sequence $\mathcal{E} / \mathbb{F}_{q^{2}}=\left(E_{k} / \mathbb{F}_{q^{2}}\right)_{k \geq 0}$, with $E_{k}=E F_{k}$, is a composite tower such that

(i) $B_{1}\left(E_{k}\right) \geq 1$ for all $k \geq 1$ and $B_{i}\left(E_{k}\right) \geq q^{k}\left(q^{2}-1\right)$ for all $i \in N$ and all $k \geq 0$

(ii) for all $k \geq 2$, one has

$$
g\left(E_{k}\right)= \begin{cases}\frac{1}{2}\left(m\left(q^{2}+2 q+2\right)-q^{2}\right) q^{k}-m(q+2) q^{\frac{k}{2}}+\frac{m+1}{2} & \text { if } k \text { even } \\ \frac{1}{2}\left(m\left(q^{2}+2 q+2\right)-q^{2}\right) q^{k}-\frac{m}{2}\left(q^{2}+3 q+1\right) q^{\frac{k-1}{2}}+\frac{m+1}{2} & \text { if } k \text { odd } .\end{cases}
$$

$$
\beta_{i}(\mathcal{E})=\frac{2\left(q^{2}-1\right)}{m\left(q^{2}+2 q+2\right)-q^{2}} \text { for all } i \in N \text { and } \beta_{i}(\mathcal{E})=0 \text { for all } i \notin N \text {. }
$$

Proof. The assertions (i) and (iii) are given in [7, Example 3.15]. We just need to prove (ii). For this we use Lemma 5.10, It is given in [7, Example 3.15] that the genus

$$
g(E)=\frac{(m-1)\left(q^{2}-1\right)}{2} .
$$

Moreover, in that example it is shown that $P_{0}$ is unramified in $E$ and $P_{\infty}$ is totally ramified in $E$. We know from [8] that

$R(\mathcal{F})=\left\{P_{0}, P_{\infty}\right\} \quad$ where $P_{0}$ (resp. $\left.P_{\infty}\right)$ is the zero (resp. the pole) of $x_{0}$ in $F_{0}$.

Hence, by using Abhyankar's Lemma and Dedekind's Different Theorem [14, we obtain that the $s$ and $r(k)$ defined in Lemma 5.10 are as follows:

$$
s=r(k)=m-1 \quad \text { for all } k \geq 0 .
$$

Now applying Lemmas 5.10 and 5.11(iii) with $r=2$, (34) and (35) give the desired result.

Proposition 5.12. The steps of the tower $\mathcal{E} / \mathbb{F}_{q^{2}}=\left(E_{k} / \mathbb{F}_{q^{2}}\right)_{k \geq 0}$ defined in Proposition 5.11 have a class number such that

$$
h\left(E_{k} / \mathbb{F}_{q^{2}}\right) \geq h_{B R T 1, k}
$$

where

$$
\begin{array}{r}
h_{B R T 1, k}=\frac{\left(q^{2}-1\right)^{2}}{\left(g\left(E_{k}\right)+1\right)\left(q^{2}+1\right)-1}\left(\prod_{i \in N \cup\{1\}}\left(\begin{array}{c}
B_{i, k}+m_{i} \\
B_{i, k}
\end{array}\right)\right. \\
+q^{2\left(g\left(E_{k}\right)-1\right)} \prod_{i \in N \cup\{1\}}\left[\left(\frac{q^{2 i}}{q^{2 i}-1}\right)^{B_{i, k}}-\right. \\
\left.\left.B_{i, k}\left(\begin{array}{c}
B_{i, k}+m_{i} \\
B_{i, k}
\end{array}\right) \int_{0}^{\frac{1}{q^{2 i}}} \frac{\left(\frac{1}{q^{2 i}}-t\right)^{m_{i}}}{(1-t)^{B_{i, k}+m_{i}+1}} \mathrm{dt} .\right]\right)
\end{array}
$$


where $B_{1, k}=1$, for all $1 \neq i \in N, B_{i, k}=q^{k}\left(q^{2}-1\right)$ and $\sum_{i \in N \cup\{1\}} i m_{i} \leq g\left(E_{k}\right)-2$ for all $k \geq 1$.

Proof. The bound $h_{B R T 1, k}$ follows directly from Theorem 4.2 by taking $l_{i}=m_{i}$ for $i \in N \cup\{1\}$ and the lower bounds for the number of places of each degree given by Proposition 5.11 .

\section{Numerical estimations:}

Here are some effective numerical estimations of the class numbers of some steps $E_{k} / \mathbb{F}_{q^{2}}$ of the tower $\mathcal{E} / \mathbb{F}_{q^{2}}$ given by Proposition 5.11 with $q=2$ and $N=\{2,3\}$. These estimations are given by using Proposition 5.12 .

\begin{tabular}{|l|c|c|c|c|c|c|c|c|r|}
\hline$q^{2}$ & step k & $g\left(E_{k}\right)$ & $B_{1, k}$ & $B_{2, k}$ & $B_{3, k}$ & $m_{1}$ & $m_{2}$ & $m_{3}$ & $h_{B R T 1, k}$ \\
\hline 4 & 2 & 55 & 1 & 12 & 12 & 13 & 11 & 6 & $3.65792120927 \times 10^{31}$ \\
\hline 4 & 3 & 132 & 1 & 24 & 24 & 29 & 25 & 17 & $9.19830033703 \times 10^{77}$ \\
\hline
\end{tabular}

5.2.2. Family 2. This family of towers is based upon the following result:

Proposition 5.13. Let $F / \mathbb{F}_{q}$ be an algebraic function field with a finite set of places $S$ and a finite separable extension $F^{\prime} / \mathbb{F}_{q}$. Further let $N \subset \mathbb{N}$ be a finite set with $m=\sum_{i \in N} i$. Suppose that $F / \mathbb{F}_{q}$ has a rational place $Q$ which has a rational extension $Q^{\prime}$ in $F^{\prime}$ such that $\left(m, e\left(Q^{\prime} \mid Q\right)\right)=1$. Define $E=F(z)$ where $z$ is a root of the polynomial

$$
\varphi(T)=\prod_{i \in N} p_{i}(T)-\alpha \in F[T]
$$

which has the following properties:

(a) for all $P \in S$, each $p_{i}(T)$ is an irreducible monic polynomial over $k(P)$ of $\operatorname{deg} p_{i}(T)=i$.

(b) $\alpha \in F$ and $\alpha(P)=0$ for all $P \in S$,

(c) $v_{Q}(\alpha)<0$ and $\left(v_{Q}(\alpha), m\right)=1$.

Set $E^{\prime}=E F^{\prime}$. Then $E^{\prime} / F$ is a separable extension such that

(i) $Q$ is totally ramified in $E,[E: F]=\left[E^{\prime}: F^{\prime}\right]=m$ and $\mathbb{F}_{q}$ is algebraically closed in $E^{\prime}$.

(ii) Each place $P \in S$ has exactly one extension $Q_{i} \in E$ with

$$
\operatorname{deg} Q_{i}=i \operatorname{deg} P \text { for all } i \in N \text {. }
$$

(iii) If $P$ splits completely in $F^{\prime}$, then each extension of $P$ in $E$ splits completely in $E^{\prime}$.

Proof. (i) By applying the generalized Eisenstein's Irreducibility Criterion [14] with the place $Q$ and using (c), we obtain that $\varphi(T)$ is irreducible over $F$ and $Q$ is totally ramified in $E$. Since $\left(m, e\left(Q^{\prime} \mid Q\right)\right)=1$ for some rational place $Q^{\prime}$ of $F^{\prime}$ lying over $Q$, it follows from Abhyankar's Lemma [14 that $Q^{\prime}$ is totally ramified in $E^{\prime}=E F^{\prime}$. Thus, (i) follows.

(ii) The proof is clear by Kummer's Theorem [14], and the properties (a) and (b).

(iii) See [14, Proposition 3.9.6(a)].

We note that a general version of Proposition 5.13 is given in 7 . 
Remark 5.14. In Proposition 5.13 the elements in the set $N$ do not need to be distinct if the following holds: for all $P \in S$ and each $i \in N$, the polynomials $p_{i}(T) \in \mathcal{O}_{P}[T]$ are pairwise distinct and irreducible over $k(P)$ with $\operatorname{deg} p(T)=i$.

\section{a) Example 1:}

Proposition 5.15. Consider the tower $\mathcal{F} / \mathbb{F}_{q}=\left(F_{k} / \mathbb{F}_{q}\right)_{k \geq 0}$ given in Proposition 5.8 with $q=2$ and the rational function field $F_{0}=\mathbb{F}_{q}\left(x_{0}\right)$. Let $E=F_{0}(z)$ where $z$ is a root of the polynomial

$$
\varphi(T)=T^{4}+T+x_{0}^{7}+1 \in F_{0}[T] .
$$

Then the sequence $\mathcal{E} / \mathbb{F}_{2}=\left(E_{k} / \mathbb{F}_{2}\right)_{k \geq 0}$, with $E_{k}=E F_{k}$, is a composite quadratic tower with the following properties:

(i) $B_{3}\left(E_{k}\right) \geq 2^{k+2}$ and $B_{6}\left(E_{k}\right) \geq 2^{k+1}$ for all $k \geq 0$,

(ii) $2^{k+3}-15 \leq g\left(E_{k}\right) \leq 7.2^{k+2}-3$ for all $k \geq 0$,

(iii) $\beta_{3}(\mathcal{E}) \geq \frac{1}{7}$ and $\beta_{6}(\mathcal{E}) \geq \frac{1}{14}$.

Proof. We first need to show that $\mathcal{E} / \mathbb{F}_{2}$ is a quadratic tower. We have that

$$
\varphi(T)=T(T+1)\left(T^{2}+T+1\right)+\left(x_{0}+1\right)\left(x_{0}^{3}+x_{0}^{2}+1\right)\left(x_{0}^{3}+x_{0}+1\right) \in F_{0}[T] .
$$

Let $k \geq 1$. We apply Proposition 5.13 and Remark 5.14 with $F, F^{\prime}=F_{k}, N=$ $\{1,1,2\}, Q=P_{\infty}=\left(x_{0}=\infty\right)$, and the set $S=\left\{Q_{1}, Q_{2}\right\} \in \mathbb{P}\left(F_{0}\right)$ with $Q_{1}:=$ $\left(x_{0}^{3}+x_{0}^{2}+1=0\right)$ and $Q_{2}:=\left(x_{0}^{3}+x_{0}+1=0\right)$. It is shown in the proof of Proposition 5.8(i) that the places $Q_{1}$ and $Q_{2}$ split completely in $E_{k}$ for all $k \geq 0$. Hence, Proposition [5.13(ii) and (iii) hold, and $\left[E: F_{0}\right]=\operatorname{deg} \varphi(T)=4$.

We now claim that $E / F_{0}$ and $F_{k} / F_{0}$ are linearly disjoint and $\mathbb{F}_{2}$ is algebraically closed in $E_{k}=E F_{k}$. Note that $\left[F_{k+1}: F_{k}\right]=2$ for all $k \geq 0$. By [6, Lemma 5.2], the place $P=\left(x_{0}=1\right)$ of $F_{0}$ is totally ramified in $F_{k}$. By Kummer's Theorem [14] $P$ is unramified in $E$ and it has an extension, say $P^{\prime}$, of degree one. Let $Q^{\prime} \in \mathbb{P}\left(E_{k}\right)$ lying above $P^{\prime}$. By Abhyankar's Lemma $e\left(Q^{\prime} \mid P^{\prime}\right)=2^{k}=\left[F_{k}: F_{0}\right] \geq\left[E_{k}: E\right]$. Since $e\left(Q^{\prime} \mid P^{\prime}\right) \leq\left[E_{k}: E\right]$, we have the equality, and so $P^{\prime}$ is totally ramified in $E_{k}$. Now the claim follows. Hence, $\mathcal{E} / \mathbb{F}_{2}$ is a tower and since $\mathcal{F} / \mathbb{F}_{2}$ is quadratic, it is also quadratic.

(i) Let $n_{i}=\#\left\{R \in \mathbb{P}(E): R \mid Q_{1}\right.$ or $R \mid Q_{2}$ with $\left.\operatorname{deg} R=i\right\}$ for $i \geq 1$. By using Proposition 5.13 (ii),(iii), we obtain that

$$
\begin{aligned}
& B_{3}\left(E_{k}\right) \geq n_{3}\left[E_{k}: E\right] \geq 4\left[E_{k}: E\right]=2^{k+2} \quad \text { and } \\
& B_{6}\left(E_{k}\right) \geq n_{6}\left[E_{k}: E\right] \geq 2\left[E_{k}: E\right]=2^{k+1} .
\end{aligned}
$$

(ii) Since the genera do not change after taking the constant field extensions, we can consider $\mathcal{E}^{\prime} / \mathbb{F}_{4}=\left(E_{k}^{\prime} / \mathbb{F}_{4}\right)_{k \geq 0}$, with $E_{k}^{\prime}:=E_{k} \mathbb{F}_{4}$, and $\mathcal{F}^{\prime} / \mathbb{F}_{4}=\left(F_{k}^{\prime} / \mathbb{F}_{4}\right)_{k \geq 0}$, with $F_{k}^{\prime}:=F_{k} \mathbb{F}_{4}$. By [14, Proposition 3.7.10], $E^{\prime} / F_{0}^{\prime}$ is an elementary Abelian extension of degree 4 . Moreover, the only ramified place in $E^{\prime} / F_{0}^{\prime}$ is the pole of $x_{0}$, say $P_{\infty}$, with

$$
e\left(Q \mid P_{\infty}\right)=4 \quad \text { and } \quad d\left(Q \mid P_{\infty}\right)=(4-1)\left(-v_{P_{\infty}}\left(x_{0}^{7}+1\right)+1\right)=24
$$

where $Q \in \mathbb{P}\left(E^{\prime}\right)$ lies above $P_{\infty}$. Hence, by the Hurwitz Genus Formula [14]

$$
g\left(E^{\prime}\right)=9 .
$$


By [7, Lemma 3.11], we have that

$$
\begin{aligned}
g\left(E_{k}\right) & \leq\left[E: F_{0}\right] g\left(F_{k}\right)+\left[F_{k}: F_{0}\right] g(E)-\left[E: F_{0}\right]\left[F_{k}: F_{0}\right] g\left(F_{0}\right) \\
& +\left(\left[E: F_{0}\right]-1\right)\left(\left[F_{k}: F_{0}\right]-1\right) \\
& =4 g\left(F_{k}\right)+12 \cdot 2^{k}-3 \quad \text { by (37) as } g(E)=g\left(E^{\prime}\right) .
\end{aligned}
$$

Moreover, one can easily conclude from the Hurwitz Genus Formula 13 for the extension $E_{k} / F_{k}$ that

$$
g\left(E_{k}\right) \geq 4 g\left(F_{k}\right)-3 .
$$

By applying Proposition 5.8 (ii) with $q=2$, we obtain that

$$
2^{k+1}-3 \leq g\left(F_{k}\right) \leq 2^{k+2} .
$$

Now combining (37), (38), (41) and (42) yields the desired bounds in (ii). (iii) follows from (i) and (ii).

Corollary 5.16. Let $\mathcal{E} / \mathbb{F}_{2}$ be the tower given in Proposition 5.15 and $\mathcal{E}^{\prime} / \mathbb{F}_{8}=$ $\left(E_{k}^{\prime} / \mathbb{F}_{8}\right)_{k \geq 0}$ with $E_{k}^{\prime}=E_{k} \mathbb{F}_{8}$. Then $\mathcal{E}^{\prime} / \mathbb{F}_{8}$ is a quadratic tower with the following properties:

(i) $B_{1}\left(E_{k}^{\prime}\right) \geq 3 \cdot 2^{k+2}$ and $B_{2}\left(E_{k}^{\prime}\right) \geq 3 \cdot 2^{k+1}$ for all $k \geq 0$.

(ii) $\beta_{1}\left(\mathcal{E}^{\prime}\right) \geq \frac{3}{7}$ and $\beta_{2}\left(\mathcal{E}^{\prime}\right) \geq \frac{3}{14}$.

Proof. The proof follows from [14, Proposition 5.1.9] and Proposition 5.15.

Proposition 5.17. The steps of the towers $\mathcal{E} / \mathbb{F}_{2}=\left(E_{k} / \mathbb{F}_{2}\right)_{k \geq 0}$ and $\mathcal{E}^{\prime} / \mathbb{F}_{8}=$ $\left(E_{k}^{\prime} / \mathbb{F}_{8}\right)_{k \geq 0}$ respectively defined in Proposition 5.15 and Corollary $[5.16$ have a class number such that

and

$$
h\left(E_{k} / \mathbb{F}_{2}\right) \geq h_{B R T 1, k}
$$

where

$$
h\left(E_{k}^{\prime} / \mathbb{F}_{8}\right) \geq h_{B R T 2, k}
$$

$$
\begin{array}{r}
h_{B R T 1, k}=\frac{1}{3\left(8.2^{k}-14\right)}\left(\prod_{i \in\{3,6\}}\left(\begin{array}{c}
B_{i, k}+m_{i} \\
B_{i, k}
\end{array}\right)\right. \\
+2^{\left(8.2^{k}-16\right)} \prod_{i \in\{3,6\}}\left[\left(\frac{2^{i}}{2^{i}-1}\right)^{B_{i, k}}-\right. \\
\left.\left.B_{i, k}\left(\begin{array}{c}
B_{i, k}+m_{i} \\
B_{i, k}
\end{array}\right) \int_{0}^{\frac{1}{2^{i}}} \frac{\left(\frac{1}{2^{i}}-t\right)^{m_{i}}}{(1-t)^{B_{i, k}+m_{i}+1}} \mathrm{dt} .\right]\right)
\end{array}
$$

and

$$
\begin{array}{r}
h_{B R T 2, k}=\frac{49}{9\left(8.2^{k}-14\right)-B_{1, k}}\left(\prod_{i \in\{1,2\}}\left(\begin{array}{c}
B_{i, k}+m_{i} \\
B_{i, k}
\end{array}\right)\right. \\
+2^{\left(8.2^{k}-16\right)} \prod_{i \in\{1,2\}}\left[\left(\frac{8^{i}}{8^{i}-1}\right)^{B_{i, k}}-\right. \\
\left.\left.B_{i, k}\left(\begin{array}{c}
B_{i, k}+m_{i} \\
B_{i, k}
\end{array}\right) \int_{0}^{\frac{1}{8^{i}}} \frac{\left(\frac{1}{8^{i}}-t\right)^{m_{i}}}{(1-t)^{B_{i, k}+m_{i}+1}} \mathrm{dt} .\right]\right)
\end{array}
$$


with

$$
B_{3, k}=2^{k+2}, B_{6, k}=2^{k+1}, B_{1, k}=3.2^{k+2}, B_{2, k}=3.2^{k+1}
$$

and

$$
3 m_{3}+6 m_{6} \leq 8.2^{k}-17
$$

and

$$
m_{1}+2 m_{2} \leq 8.2^{k}-17
$$

for all $k \geq 1$.

Proof. The bound $h_{B R T 1, k}$ follows directly from Theorem 4.2 by taking $l_{i}=m_{i}$ for $i=3,6$ and the lower bounds for the number of places of each degree given by Proposition 5.15. The bound $h_{B R T 2, k}$ follows directly from Theorem 4.2 by taking $l_{i}=m_{i}$ for $i=1,2$ and the lower bounds for the number of places of each degree given by Corollary 5.16 .

\section{Numerical estimations:}

Here are some effective numerical estimations of the class numbers of some steps $E_{k} / \mathbb{F}_{2}$ and $E_{k}^{\prime} / \mathbb{F}_{8}$ of respectively the towers $\mathcal{E} / \mathbb{F}_{2}$ and $\mathcal{E}^{\prime} / \mathbb{F}_{8}$ given by Proposition 5.15. These estimations are given by using Proposition 5.17

\begin{tabular}{|c|c|c|c|c|c|c|c|}
\hline $\mathrm{q}$ & step k & $g_{k}$ & $B_{3, k}$ & $B_{6, k}$ & $m_{3}$ & $m_{6}$ & $h_{B R T 1, k}$ \\
\hline 2 & 2 & 17 & 16 & 8 & 5 & 0 & 10254 \\
\hline 2 & 3 & 49 & 32 & 16 & 11 & 2 & $1.71832288189 \times 10^{14}$ \\
\hline
\end{tabular}

\begin{tabular}{|l|c|c|c|c|c|c|c|}
\hline$q^{3}$ & step k & $g_{k}$ & $B_{1, k}$ & $B_{2, k}$ & $m_{1}$ & $m_{2}$ & $h_{B R T 2, k}$ \\
\hline 8 & 2 & 17 & 48 & 24 & 11 & 2 & $1.0021797677 \times 10^{17}$ \\
\hline 8 & 3 & 49 & 96 & 48 & 33 & 7 & $2.42674161125 \times 10^{48}$ \\
\hline
\end{tabular}

\section{b) Example 2:}

Proposition 5.18. Let $N \subset \mathbb{N}$ be a finite set and set $m=\sum_{i \in N} i$. Let $l$ be $a$ prime power, $q=l^{r}$ with $r \geq 2$, and $d=\frac{q-1}{l-1}$. Suppose that $(m, d q)=1$. Further let $\mathcal{F} / \mathbb{F}_{q}=\left(F_{k} / \mathbb{F}_{q}\right)_{k \geq 0}$, with the rational function field $F_{0}=\mathbb{F}_{q}\left(x_{0}\right)$, be the tower which is recursively defined by the polynomial

$$
f(X, Y)=Y^{d}-a(X+b)^{d}+c \in \mathbb{F}_{q}[X, Y]
$$

with $a, c \in \mathbb{F}_{l}, b \in \mathbb{F}_{q}^{*}$ and $a b^{d}+c=0$. Let $E=F_{0}(z)$ with $z$ a root of the polynomial

$$
\varphi(T)=\prod_{i \in N} p_{i}(T)-\frac{1}{x_{0}} \in F_{0}[T],
$$

where each $p_{i}(T) \in \mathbb{F}_{q}[T]$ is an irreducible monic polynomial of $\operatorname{deg} p_{i}(T)=i$. Suppose that $\left(\varphi(T), \varphi^{\prime}(T)\right)=1$ at the places $P \in R(\mathcal{F}) \backslash\left\{P_{0}\right\}$ and $\varphi(T)$ is separable. Then the sequence $\mathcal{E} / \mathbb{F}_{q}=\left(E_{k} / \mathbb{F}_{q}\right)_{k \geq 0}$, with $E_{k}:=E F_{k}$, is a composite tower such that

(i) $B_{1}\left(E_{k}\right) \geq 1$ and $B_{i}\left(E_{k}\right) \geq d^{k}$ for all $i \in N$ and $k \geq 0$,

(ii) for all $k \geq 1$

$$
\left(\frac{m q-m-2}{4}\right) d^{k}+\frac{1}{2} \leq g\left(E_{k}\right) \leq\left(\frac{m q-m-1}{2}\right) d^{k}+\frac{(1-q) m+1}{2} .
$$

(iii) $\beta_{i}(\mathcal{E}) \geq \frac{2}{m q-m-1}$ for all $i \in N$. 
Proof. By [22, Theorem 4.2.3 and Lemma 4.2.2], the tower $\mathcal{F} / \mathbb{F}_{q}$ has the following properties:

(a) the pole (resp. the zero) of $x_{0}$, say $P_{\infty}$ (resp. $P_{0}$ ) splits completely (resp. totally ramified) in $\mathcal{F} / \mathbb{F}_{q}$,

(b) $\beta_{1}(\mathcal{F})=\frac{2}{q-2}$,

(c) for any $k \geq 1$, the genus

$$
\begin{aligned}
g\left(F_{k}\right)=\left(\frac{q-2}{2}\right) d^{k}- & \frac{1}{2} a_{k}+1 \text { with } \lim _{k \rightarrow \infty} \frac{a_{k}}{\left[F_{k}: F_{0}\right]}=0, \text { where } \\
a_{k} & :=\sum_{P \in R(\mathcal{F})} \sum_{\substack{Q \in \mathbb{P}\left(F_{k}\right) \\
Q \mid P}} \operatorname{deg} Q .
\end{aligned}
$$

(d) $R(\mathcal{F})=\left\{\left(x_{0}-\alpha\right) \in \mathbb{P}\left(F_{0}\right) \mid \alpha \in \mathbb{F}_{q}\right\}$.

By applying Proposition 5.13 with the set $S=\left\{P_{\infty}\right\}$ and $Q=P_{0}$, we obtain that the sequence $\mathcal{E} / \mathbb{F}_{q}=\left(E_{k} / \mathbb{F}_{q}\right)_{k \geq 0}$ is a tower with the following properties:

$\left(^{*}\right)\left[E: F_{0}\right]=\left[E_{k}: F_{k}\right]=\operatorname{deg} \varphi(T)=m$ for all $k \geq 0$, (observe that then $E / F$ and $F_{k} / F$ are linearly disjoint for all $\left.k \geq 0\right)$.

(**) for each $i \in N$, the place $P_{\infty}$ has exactly one extension $Q_{i}$ in $E$ with $\operatorname{deg} Q_{i}=i$ and $Q_{i}$ splits completely in $\mathcal{E}$.

By (a), Proposition 5.13(i), and Abhyankar's Lemma[14, $P_{0}$ is totally ramified in $E_{k}$ for all $k \geq 0$. Hence, $E_{k}$ has an $\mathbb{F}_{q}$-rational place, namely the extension of $P_{0}$. Thus, $B_{1}\left(E_{k}\right) \geq 1$. The second part of (i) is clear by $\left(^{*}\right)$ and $(* *)$.

(ii) We will use Lemma [5.10, It is clear that $g(E)=0$. Let $P \in \mathcal{R}(\mathcal{F}) \backslash\left\{P_{0}\right\}$. By assumption $\left(\varphi(T), \varphi^{\prime}(T)\right)=1$ at $P$, and so $\varphi(T)$ has no multiple factors over the residue class field of $P$. Thus, by Kummer's Theorem [14, $P$ is unramified in $E$. By Abhyankar's Lemma [14, any extension of $P$ in $F_{k}$ is then unramified in $E_{k}$ for all $k \geq 1$. Thus, the $s$ and $r(k)$ defined in Lemma 5.10 are as follows:

$$
s=r(k)=m-1 \text { for all } k \geq 1 .
$$

Now it follows from Lemma 5.10 and (c) that

$$
g\left(E_{k}\right)=\left(\frac{m q-m-1}{2}\right) d^{k}-\frac{m}{2} a_{k}+\frac{m+1}{2} \text { with } \lim _{k \rightarrow \infty} \frac{a_{k}}{\left[E_{k}: E\right]}=0 .
$$

Next, we want to estimate $a_{k}$. By (a), (d) and the fact that $e(Q \mid P) \geq 2$ and $\operatorname{deg} P=1$ for all $P \in R(\mathcal{F})$, we obtain that

$$
\begin{aligned}
a_{k} & =\sum_{P \in R(\mathcal{F})} \sum_{\substack{Q \in \mathbb{P}\left(F_{k}\right) \\
Q \mid P}} \operatorname{deg} Q=\sum_{P \in R(\mathcal{F})} \sum_{\substack{Q \in \mathbb{P}\left(F_{k}\right) \\
Q \mid P}} f(Q \mid P) \operatorname{deg} P \\
= & \sum_{P \in R(\mathcal{F}) \backslash\left\{P_{0}\right\}} \sum_{Q \in \mathbb{P}\left(F_{k}\right)} \operatorname{deg} Q+\sum_{Q \in \mathbb{P}\left(F_{k}\right)} f\left(Q \mid P_{0}\right) \operatorname{deg} P_{0} \\
= & \sum_{P \in R(\mathcal{F}) \backslash\left\{P_{0}\right\}} \sum_{\substack{Q \in \mathbb{P}\left(F_{k}\right) \\
Q \mid P}} f(Q \mid P) \operatorname{deg} P+1 \\
\leq & \sum_{P \in R(\mathcal{F}) \backslash\left\{P_{0}\right\}} \sum_{\substack{Q \in \mathbb{P}\left(F_{k}\right) \\
Q \mid P}} 2 f(Q \mid P)+1 \\
\leq & \sum_{P \in R(\mathcal{F}) \backslash\left\{P_{0}\right\}} \sum_{\substack{Q \in \mathbb{P}\left(F_{k}\right) \\
Q \mid P}} e(Q \mid P) f(Q \mid P)+1 \\
\leq & (\# R(\mathcal{F})-1)\left[F_{k}: F_{0}\right]+1=(q-1) d^{k}+1, \\
& \text { by Fundamental Equality } 14 .
\end{aligned}
$$


Hence, by (46),

$$
a_{k}=\sum_{P \in R(\mathcal{F}) \backslash\left\{P_{0}\right\}} \sum_{\substack{Q \in \mathbb{P}\left(F_{k}\right) \\ Q \mid P}} f(Q \mid P) \operatorname{deg} P+1 \leq \frac{(q-1)}{2} d^{k}+1
$$

On the other hand as $f(Q \mid P) \geq 1$, by (46),

$$
a_{k} \geq(\# R(\mathcal{F})-1)+1=q
$$

Therefore, by (47) and (48),

$$
q \leq a_{k} \leq \frac{(q-1)}{2} d^{k}+1
$$

Now (ii) follows from (45) and (49). (iii) follows from (i) and (ii).

Proposition 5.19. The steps of the tower $\mathcal{E} / \mathbb{F}_{q}=\left(E_{k} / \mathbb{F}_{q}\right)_{k \geq 0}$ defined in Proposition 5.18 have a class number such that

$$
h\left(E_{k} / \mathbb{F}_{q}\right) \geq h_{B R T 1, k}
$$

where

$$
\begin{array}{r}
h_{B R T 1, k}=\frac{(q-1)^{2}}{\left(g_{k}+1\right)(q+1)-1}\left(\prod_{i \in N \cup\{1\}}\left(\begin{array}{c}
B_{i, k}+m_{i} \\
B_{i, k}
\end{array}\right)\right. \\
+q^{g_{k}-1} \prod_{i \in N \cup\{1\}}\left[\left(\frac{q^{i}}{q^{i}-1}\right)^{B_{i, k}}-\right. \\
\left.\left.B_{i, k}\left(\begin{array}{c}
B_{i, k}+m_{i} \\
B_{i, k}
\end{array}\right) \int_{0}^{\frac{1}{q^{i}}} \frac{\left(\frac{1}{q^{i}}-t\right)^{m_{i}}}{(1-t)^{B_{i, k}+m_{i}+1}} \mathrm{dt} .\right]\right)
\end{array}
$$

where $B_{i, k}=d^{k}$ for all $i \in N, g_{k}=\left\lceil\left(\frac{m q-m-2}{4}\right) d^{k}+\frac{1}{2}\right\rceil$ and $\sum_{i \in N \cup\{1\}} i m_{i} \leq g_{k}-2$ for all $k \geq 1$.

Proof. The bound $h_{B R T 1, k}$ follows directly from Theorem 4.2 by taking $l_{i}=m_{i}$ for $i \in N \cup\{1\}$ and the lower bounds for the genus and for the number of places of each degree given by Proposition 5.18 .

\section{Numerical estimations:}

Here are some effective numerical estimations of the class numbers of some steps $E_{k} / \mathbb{F}_{q}$ of the tower $\mathcal{E} / \mathbb{F}_{q}$ given by Proposition 5.18 with $q=4, d=3$, and $N=$ $\{2,3\}$. These estimations are given by using Proposition 5.18 ,

\begin{tabular}{|c|c|c|c|c|c|c|c|c|r|}
\hline$q$ & step k & $g\left(E_{k}\right)$ & $B_{1, k}$ & $B_{2, k}$ & $B_{3, k}$ & $m_{1}$ & $m_{2}$ & $m_{3}$ & $h_{B R T 1, k}$ \\
\hline 4 & 2 & 30 & 1 & 9 & 9 & 7 & 6 & 3 & $4.625820113117 \times 10^{16}$ \\
\hline 4 & 3 & 89 & 1 & 27 & 27 & 18 & 18 & 11 & $2.23693900061 \times 10^{52}$ \\
\hline
\end{tabular}

c) Example 3:

Proposition 5.20. Let $\mathcal{F} / \mathbb{F}_{9}=\left(F_{k} / \mathbb{F}_{9}\right)_{k \geq 0}$ be the tower defined by the polynomial

$$
f(X, Y)=Y^{2}+(X+b)^{2}-1 \in \mathbb{F}_{9}[X, Y] \quad \text { with } \quad b \in \mathbb{F}_{l}^{*}
$$


and $F_{0}=\mathbb{F}_{9}\left(x_{0}\right)$ be the rational function field. Let $E=F_{0}(z)$ with $z$ a root of the polynomial

$$
\varphi(T)=\left(T^{2}+\mu^{7}\right)\left(T^{9}-T\right)-\frac{1}{x_{0}} \in F_{0}[T],
$$

where $\mu$ is a primitive element for $\mathbb{F}_{9}$. Then the sequence $\mathcal{E} / \mathbb{F}_{9}=\left(E_{k} / \mathbb{F}_{9}\right)_{k \geq 0}$, with $E_{k}=E F_{k}$, is a composite quadratic tower such that

(i) $B_{1}\left(E_{k}\right) \geq 9 \cdot 2^{k}$ and $B_{2}\left(E_{k}\right) \geq 2^{k}$ for all $k \geq 0$.

(ii) for all $k \geq 0$,

$$
g\left(E_{k}\right)=\left\{\begin{array}{l}
21 \cdot 2^{k-1}-33 \cdot 2^{(k-2) / 2}+6 \quad \text { if } k \equiv 0 \quad \bmod 2 \\
21 \cdot 2^{k-1}-11 \cdot 2^{(k+1) / 2}+6 \quad \text { if } k \equiv 1 \quad \bmod 2 .
\end{array}\right.
$$

(iii) $\beta_{1}\left(\mathcal{E} / \mathbb{F}_{9}\right)=\frac{6}{7}, \beta_{2}\left(\mathcal{E} / \mathbb{F}_{9}\right)=\frac{2}{21}$ and $\beta_{r}\left(\mathcal{E} / \mathbb{F}_{9}\right)=0$ for all $r \neq 1,2$.

Proof. By [9, Theorem 3.11] and [19, Proposition 3.3.12], the tower $\mathcal{F} / \mathbb{F}_{4}$ has the following properties:

(a) $\mathcal{R}(\mathcal{F})=\left\{P \in \mathbb{P}\left(F_{0}\right): x_{0}(P)=\alpha\right.$ for some $\left.\alpha \in \mathbb{F}_{3}\right\}$.

(b) The pole (resp. the zero) of $x_{0}$, say $P_{\infty}$ (resp. $P_{0}$ ), splits completely (resp. is totally ramified) in $\mathcal{F} / \mathbb{F}_{q}$.

(c) For all $k \geq 0$,

$$
g\left(F_{k}\right)= \begin{cases}2^{k-1}-3 \cdot 2^{(k-2) / 2}+1 & \text { if } k \equiv 0 \quad \bmod 2 \\ 2^{k-1}-2^{(k+1) / 2}+1 & \text { if } k \equiv 1 \bmod 2,\end{cases}
$$

(d) $\mathcal{F} / \mathbb{F}_{9}$ is maximal with $\beta_{1}\left(\mathcal{F} / \mathbb{F}_{9}\right)=2$.

By applying Proposition 5.13 with the place $Q=P_{0}$ and $S=\left\{P_{\infty}\right\}$, we obtain that the sequence $\mathcal{E} / \mathbb{F}_{9}$ is a quadratic tower and the assertion (i) holds.

(ii) We will again apply Lemma5.10. One can easily check that $\left(\varphi(T), \varphi^{\prime}(T)\right)=1$ at the places $P \in \mathbb{P}(F)$ with $x_{0}(P)=\alpha$ for some $\alpha \in \mathbb{F}_{3}^{*}$. Hence, $\varphi(T)$ has no multiple factor over the residue class field of these places. Thus, it follows from Kummer's Theorem [14] that these places are unramified in E. Then by Dedekind's Different Theorem [14], the $s$ in Lemma 5.10 is

$$
s=10 \quad \text { where } Q_{0} \text { is the extension of } P_{0} \text { in } E .
$$

By using Abhyankar's Lemma [14, we have that all $P_{k} \in \mathbb{P}\left(F_{k}\right)$ with $P_{k} \cap F \in\{P \in$ $\left.\mathbb{P}(F): x_{0}(P) \in \mathbb{F}_{3}^{*}\right\}$ are unramified in $E_{k}$. Moreover, since $P_{0}$ is totally ramified in both extensions $E / F_{0}$ and $F_{k} / F_{0}$, again by the same lemma, we have that any extension of $P_{0}$ in $F_{k}$ is totally ramified in $E_{k}$. Hence,

$$
r(k)=10 \text { for all } k \geq 1 .
$$

Now by applying Lemma 5.10, (51), (52), and (c), we obtain the desired result. (iii) follows from (i), (ii), (d) and [7, Theorem 3.7(ii)].

Proposition 5.21. The steps of the tower $\mathcal{E} / \mathbb{F}_{9}=\left(E_{k} / \mathbb{F}_{9}\right)_{k \geq 0}$ defined in Proposition 5.20 have a class number such that

$$
h\left(E_{k} / \mathbb{F}_{9}\right) \geq h_{B R T 1, k}
$$


where

$$
\begin{array}{r}
h_{B R T 1, k}=\frac{64}{10\left(g_{k}+1\right)-9.2^{k}}\left(\prod_{i \in\{1,2\}}\left(\begin{array}{c}
B_{i, k}+m_{i} \\
B_{i, k}
\end{array}\right)\right. \\
+9^{g\left(E_{k}\right)-1} \prod_{i \in\{1,2\}}\left[\left(\frac{9^{i}}{9^{i}-1}\right)^{B_{i, k}}-\right. \\
\left.\left.B_{i, k}\left(\begin{array}{c}
B_{i, k}+m_{i} \\
B_{i, k}
\end{array}\right) \int_{0}^{\frac{1}{9^{i}}} \frac{\left(\frac{1}{9^{i}}-t\right)^{m_{i}}}{(1-t)^{B_{i, k}+m_{i}+1}} \mathrm{dt} .\right]\right)
\end{array}
$$

where $B_{1, k}=9.2^{k}, B_{2, k}=2^{k}$, and $m_{1}+2 m_{2} \leq g\left(E_{k}\right)-2$ for all $k \geq 1$.

Proof. The bound $h_{B R T 1, k}$ follows directly from Theorem 4.2 by taking $l_{i}=m_{i}$ for $i \in\{1,2\}$ and the lower bounds for the number of places of each degree given by Proposition 5.20 .

\section{Numerical estimations:}

Here are some effective numerical estimations of the class numbers of some steps $E_{k} / \mathbb{F}_{q}$ of the tower $\mathcal{E} / \mathbb{F}_{q}$ given by Proposition 5.20 . These estimations are given by using Proposition 5.21 .

\begin{tabular}{|c|c|c|c|c|c|c|c|}
\hline$q$ & step k & $g_{k}$ & $B_{1, k}$ & $B_{2, k}$ & $m_{1}$ & $m_{2}$ & $h_{B R T 1, k}$ \\
\hline 9 & 2 & 15 & 36 & 4 & 11 & 1 & $8.56396791978 \times 10^{14}$ \\
\hline 9 & 3 & 46 & 72 & 8 & 34 & 5 & $7.47053482878 \times 10^{45}$ \\
\hline
\end{tabular}

\section{d) Example 4:}

Proposition 5.22. Let $\mathcal{F} / \mathbb{F}_{4}=\left(F_{k} / \mathbb{F}_{4}\right)_{k \geq 0}$ be the tower defined by the polynomial

$$
f(X, Y)=Y^{3}+(X+1)^{3}-1 \in \mathbb{F}_{4}[X, Y]
$$

and $F_{0}=\mathbb{F}_{4}\left(x_{0}\right)$ be the rational function field. Let $E=F_{0}(z)$ with $z$ a root of the polynomial

$$
\varphi(T)=\left(T^{4}-T\right)\left(T^{2}+\mu T+\mu\right)-\frac{1}{x_{0}} \in F_{0}[T],
$$

where $\mu$ is a primitive element for $\mathbb{F}_{4}$.

Then the sequence $\mathcal{E} / \mathbb{F}_{4}=\left(E_{k} / \mathbb{F}_{4}\right)_{k \geq 0}$, with $E_{k}=E F_{k}$, is a composite tower such that

(i) $B_{1}\left(E_{k}\right) \geq 4 \cdot 3^{k}$ and $B_{2}\left(E_{k}\right) \geq 3^{k}$ for all $k \geq 0$,

(ii) $g\left(E_{k}\right)= \begin{cases}\frac{13}{2} \cdot 3^{k}-4 \cdot 3^{(k+2) / 2}+\frac{5}{2} & \text { if } k \equiv 0 \bmod 2 \\ \frac{13}{2} \cdot 3^{k}-2 \cdot 3^{(k+3) / 2}+\frac{5}{2} & \text { if } k \equiv 1 \bmod 2 .\end{cases}$

(iii) $\beta_{1}\left(\mathcal{E} / \mathbb{F}_{4}\right)=\frac{8}{13}, \beta_{2}\left(\mathcal{E} / \mathbb{F}_{4}\right)=\frac{2}{13}$ and $\beta_{r}\left(\mathcal{E} / \mathbb{F}_{4}\right)=0$ for all $r \neq 1,2$.

Proof. By [9, Theorem 3.11] and [19, Proposition 3.3.12], the tower $\mathcal{F} / \mathbb{F}_{4}$ has the following properties:

(a) $\mathcal{R}(\mathcal{F})=\left\{P \in \mathbb{P}\left(F_{0}\right): x_{0}(P)=\alpha\right.$ for some $\left.\alpha \in \mathbb{F}_{4}\right\}$.

(b) The pole (resp. the zero) of $x_{0}$, say $P_{\infty}$ (resp. $P_{0}$ ), splits completely (resp. is totally ramified) in $\mathcal{F} / \mathbb{F}_{q}$. 
(c) For all $k \geq 1$,

$$
g\left(F_{k}\right)=\left\{\begin{array}{lll}
3^{k}-2 \cdot 3^{k / 2}+1 & \text { if } k \equiv 0 & \bmod 2 \\
3^{k}-3^{(k+1) / 2}+1 & \text { if } k \equiv 1 & \bmod 2
\end{array}\right.
$$

(d) The tower $\mathcal{F} / \mathbb{F}_{4}$ is maximal with $\beta_{1}\left(\mathcal{F} / \mathbb{F}_{4}\right)=1$.

By applying Kummer's Theorem [14] and Proposition 5.13 with the set $S=\left\{P_{\infty}\right\}$ and $Q=P_{0}$, we obtain the following:

(1) $\left[E: F_{0}\right]=\operatorname{deg} \varphi(T)=6$ for all $k \geq 0$,

(2) $P_{0}$ is totally ramified in $E$, and so $\mathbb{F}_{4}$ is algebraically closed in $E$,

(3) $P_{\infty}$ has four rational extensions and one extension of degree two in $E$, and they all split completely in $E_{k}$ for all $k \geq 1$,

(4) Since $\left(\varphi(T), \varphi^{\prime}(T)\right)=1$ at the places $P_{\mu}=\left(x_{0}=\mu\right)$ and $P_{\mu^{2}}=\left(x_{0}=\mu^{2}\right)$, these places are unramified in $E$.

(5) $P_{1}=\left(x_{0}=1\right)$ has exactly one extension which has degree 2 and ramification index 3 (by using Magma).

By (3), $E_{k} / \mathbb{F}_{4}$ has an $\mathbb{F}_{4}$-rational place, and so $\mathbb{F}_{4}$ is algebraically closed in $E_{k}$ for all $k \geq 0$. By [19, Lemma 3.3.9(i)], the place $\left(x_{0}=\mu^{2}\right)$ is totally ramified in $F_{k}$. Let $Q$ be an extension of $\left(x_{0}=\mu^{2}\right)$ in $E$. Then using (4) and Abhyankar's Lemma 14 gives that $Q$ has an extension, say $Q^{\prime}$ in $E_{k}$ with the ramification index such that

$$
\left[E_{k}: E\right] \geq e\left(Q^{\prime} \mid Q\right)=\left[F_{k}: F_{0}\right] \geq\left[E_{k}: E\right] .
$$

Thus, $\left[E_{k}: E\right]=\left[F_{k}: F_{0}\right]$, i.e., $E_{k} / F_{0}$ and $F_{k} / F_{0}$ are linearly disjoint. Consequently, the sequence $\mathcal{E} / \mathbb{F}_{4}$ is a composite tower.

(i) follows from (3):

$$
B_{1}\left(E_{k}\right) \geq 4 \cdot\left[E_{k}: E\right]=4 \cdot 3^{k} \text { and } B_{2}\left(E_{k}\right) \geq\left[E_{k}: E\right]=3^{k} \text { for all } k \geq 0 .
$$

(ii) We will use the Hurwitz Genus Formula [13 for the extension $E_{k} / F_{k}$ for any $k \geq 1$. It is clear that $g(E)=0$. By applying the Hurwitz Genus Formula for the extension $E / F_{0}$, we get that the degree of the different of $E / F_{0}$ is

$$
\operatorname{deg} \operatorname{Diff}\left(E / F_{0}\right)=10 \text {. }
$$

Let $Q \in \mathbb{P}(E)$. By using (2), (5), and Dedekind's Different Formula [14, we have that

$\left(^{*}\right)$ if $Q \mid P_{1}$, then $d\left(Q \mid P_{1}\right)=e\left(Q \mid P_{1}\right)-1=2$.

${ }^{(* *)}$ If $Q \mid P_{0}$, then $d\left(Q \mid P_{0}\right)>e\left(Q \mid P_{0}\right)-1=5$. Moreover, by (*), (5), and (55), we have that $6 \geq d\left(Q \mid P_{0}\right)$. Hence, $d\left(Q \mid P_{0}\right)=6$.

Notice that (55), $\left({ }^{*}\right)$, and $\left(^{* *}\right)$ then imply that only $P_{0}$ and $P_{1}$ are ramified in $E / F_{0}$. Now let $P_{k}$ be a place of $F_{k}, P:=P_{k} \cap F_{0}$ and $Q_{k}$ be an extension of $P_{k}$ in $E_{k}$. Set $Q:=Q_{k} \cap E$. Then clearly $Q \mid P$. By Abhyankar's Lemma [13, if $e\left(Q_{k} \mid P_{k}\right)>1$, then $e(Q \mid P)>1$. Hence, it is enough to consider just the places $P_{0}$ and $P_{1}$. First suppose that $P:=P_{1}$. By [7, Lemma 3.3.9], $P$ is totally ramified in $F_{k}$. Thus, using (5) and Abhyankar's lemma, we get that $e\left(Q_{k} \mid P_{k}\right)=1$.

Now suppose that $P:=P_{0}$. By (b), (2) and Abhyankar's Lemma, we have that

$$
e\left(Q_{k} \mid P_{k}\right)=2 \quad \text { and } \quad e\left(Q_{k} \mid Q\right)=3^{k-1} \text {. }
$$

Thus, by Dedekind's Different Theorem [13]

$$
d\left(Q_{k} \mid Q\right)=e\left(Q_{k} \mid Q\right)-1=3^{k-1}-1 \quad \text { and } \quad d\left(P_{k} \mid P\right)=e\left(P_{k} \mid P\right)-1=3^{k}-1 .
$$


Now using (**), (56), (57) and applying Transitivity of Different [13] in $F_{0} \subseteq E \subseteq$ $E_{k}$ and $F_{0} \subseteq F_{k} \subseteq E_{k}$, we obtain that

$$
\begin{gathered}
e\left(Q_{k} \mid P_{k}\right) d\left(P_{k} \mid P\right)+d\left(Q_{k} \mid P_{k}\right)=2\left(3^{k}-1\right)+d\left(Q_{k} \mid P_{k}\right) \\
=e\left(Q_{k} \mid Q\right) d(Q \mid P)+d\left(Q_{k} \mid Q\right)=6 \cdot 3^{k-1}+3^{k-1}-1 .
\end{gathered}
$$

Hence,

$$
d\left(Q_{k} \mid P_{k}\right)=3^{k-1}+1
$$

Now using (56), (58), and the Hurwitz Genus Formula for the extension $E_{k} / F_{k}$, we obtain that

$$
\begin{aligned}
2 g\left(E_{k}\right)-2 & \left.=6\left(2 g\left(F_{k}\right)-2\right)+\operatorname{deg} \operatorname{Diff}\left(E_{k} / F_{k}\right)\right) \\
& =6\left(2 g\left(F_{k}\right)-2\right)+\sum_{Q_{k} \mid P_{k}} d\left(Q_{k} \mid P_{k}\right) \operatorname{deg}\left(Q_{k}\right) \\
& =6\left(2 g\left(F_{k}\right)-2\right)+\sum_{Q_{k} \mid P_{k}}\left(3^{k-1}+1\right) f\left(Q_{k} \mid P_{k}\right) \\
& =6\left(2 g\left(F_{k}\right)-2\right)+3 \cdot\left(3^{k-1}+1\right) \quad \text { by Fundamental Equality [13] } \\
& =12 g\left(F_{k}\right)-12+3^{k}+3 .
\end{aligned}
$$

Hence,

$$
g\left(E_{k}\right)=6 g\left(F_{k}\right)+\frac{3^{k}}{2}-\frac{7}{2} .
$$

Now, combining this with (c) yields the desired result for $g\left(E_{k}\right)$.

(iii) follows from (i), (ii), (d) and 7, Theorem 3.7(ii)].

Proposition 5.23. The steps of the tower $\mathcal{E} / \mathbb{F}_{4}=\left(E_{k} / \mathbb{F}_{4}\right)_{k \geq 0}$ defined in Proposition 5.20 have a class number such that

$$
h\left(E_{k} / \mathbb{F}_{4}\right) \geq h_{B R T 1, k}
$$

where

$$
\begin{array}{r}
h_{B R T 1, k}=\frac{9}{5\left(g_{k}+1\right)-4.3^{k}}\left(\prod_{i \in\{1,2\}}\left(\begin{array}{c}
B_{i, k}+m_{i} \\
B_{i, k}
\end{array}\right)\right. \\
+4^{g\left(E_{k}\right)-1} \prod_{i \in\{1,2\}}\left[\left(\frac{4^{i}}{4^{i}-1}\right)^{B_{i, k}}-\right. \\
\left.\left.B_{i, k}\left(\begin{array}{c}
B_{i, k}+m_{i} \\
B_{i, k}
\end{array}\right) \int_{0}^{\frac{1}{4^{i}}} \frac{\left(\frac{1}{4^{i}}-t\right)^{m_{i}}}{(1-t)^{B_{i, k}+m_{i}+1}} \mathrm{dt} .\right]\right)
\end{array}
$$

where $B_{1, k}=4.3^{k}, B_{2, k}=3^{k}$, and $m_{1}+2 m_{2} \leq g\left(E_{k}\right)-2$ for all $k \geq 1$.

Proof. The bound $h_{B R T 1, k}$ follows directly from Theorem 4.2 by taking $l_{i}=m_{i}$ for $i \in\{1,2\}$ and the lower bounds for the number of places of each degree given by Proposition 5.22 .

\section{Numerical estimations:}

Here are some effective numerical estimations of the class numbers of some steps $E_{k} / \mathbb{F}_{q}$ of the tower $\mathcal{E} / \mathbb{F}_{q}$ given by Proposition 5.22 . These estimations are given by using Proposition 5.23 . 


\begin{tabular}{|c|c|c|c|c|c|c|c|}
\hline$q$ & step k & $g_{k}$ & $B_{1, k}$ & $B_{2, k}$ & $m_{1}$ & $m_{2}$ & $h_{B R T 1, k}$ \\
\hline 4 & 2 & 25 & 36 & 9 & 19 & 2 & $1.41572226696 \times 10^{18}$ \\
\hline 4 & 3 & 124 & 108 & 27 & 90 & 16 & $3.50178913096 \times 10^{86}$ \\
\hline
\end{tabular}

\section{3. infinitely many.}

Proposition 5.24. Let $\mathbb{F}_{q}$ be a finite field of characteristic $p$ and $m \in \mathbb{N}$ with $(m, p)=1$ and $m \geq 2$. Then there is a tower $\mathcal{F} / \mathbb{F}_{q}=\left(F_{k} / \mathbb{F}_{q}\right)_{k \geq 0}$ and a strictly increasing sequence $\left(n_{i}\right)_{i \geq 0}$ of positive integers with $n_{0}=1$ such that

(i) $B_{n_{i}}\left(F_{k}\right) \geq p^{k-i}$ for all $0 \leq i \leq k$,

(ii) $g\left(F_{k}\right) \geq \frac{m-1}{2}\left(p^{k}-1\right)$ for all $k \geq 0$.

Proof. (i) The proof is similar to that of [7, Lemma 3.17]. Let $F_{0}=\mathbb{F}_{q}\left(x_{0}\right)$ be the rational function field. Set

$$
Q_{0}=\left(x_{0}=\infty\right), S_{0}=\left\{P_{0}\right\} \text { where } P_{0}=\left(x_{0}=0\right) \text {, and } n_{=} 1 .
$$

Choose an element $z_{0} \in F_{0}$ with the following properties:

$$
z_{0}(P)=0 \text { for } P \in S_{0} \text { and } v_{Q_{0}}\left(z_{0}\right)=-m \text {. }
$$

Note that by the Weak Approximation Theorem [14] such an element $z_{0}$ always exists. Let $F_{1}=F_{0}\left(x_{1}\right)$ where $x_{1}$ satisfies the equation

$$
x_{1}^{p}-x_{1}=z_{0} .
$$

Then $Q_{0}$ is totally ramified in $F_{1}$, and hence $\mathbb{F}_{q}$ is algebraically closed in $F_{1}$. Denote by $Q_{1}$ the place of $F_{1}$ lying above $Q_{0}$. Then by Artin-Schreier Extension Theorem [14,

Set

$$
d\left(Q_{1} \mid Q_{0}\right)=(m+1)(p-1) .
$$

$$
S_{1}=\left\{P \in \mathbb{P}\left(F_{1}\right): P \cap F_{0} \in S_{0}\right\} \cup\left\{P_{1}\right\},
$$

where $P_{1} \in \mathbb{P}\left(F_{1}\right)$ having $\operatorname{deg} P_{1}=n_{1}$ for some $n_{1}>1$. Next, choose an element $z_{1} \in F_{1}$ such that

$$
z_{1}(P)=0 \text { for all } P \in S_{1} \text { and } v_{Q_{1}}\left(z_{1}\right)=-m
$$

Let $F_{2}=F_{1}\left(x_{2}\right)$ where $x_{2}$ satisfies the equation

$$
x_{2}^{p}-x_{2}=z_{1} .
$$

Then $Q_{1}$ is totally ramified in $F_{2}$, and so $\mathbb{F}_{q}$ is algebraically closed in $F_{2}$. Moreover, again by Artin-Schreier Extension Theorem [14],

$$
d\left(Q_{2} \mid Q_{1}\right)=(m+1)(p-1),
$$

where $Q_{2} \in \mathbb{P}\left(F_{2}\right)$ lies above $Q_{1}$. We continue on this process inductively for $k \geq 2$. Set

$$
S_{k-1}=\left\{P \in \mathbb{P}\left(F_{k-1}\right): P \cap F_{k-2} \in S_{k-2}\right\} \cup\left\{P_{k-1}\right\}
$$

where $P_{k-1} \in \mathbb{P}\left(F_{k-1}\right)$ of $\operatorname{deg} P_{k-1}=n_{k-1}$ for some $n_{k-1}>n_{k-2}$. Choose an element $z_{k-1} \in F_{k-1}$ such that the following hold:

$$
z_{k-1}(P)=0 \text { for all } P \in S_{k-1} \text { and } v_{Q_{k-1}}\left(z_{k-1}\right)=-m
$$

where $Q_{k-1} \in \mathbb{P}\left(F_{k-1}\right)$ lies above $Q_{k-2}$. Then $Q_{k-1}$ is totally ramified in $F_{k}$, and hence $\mathbb{F}_{q}$ is algebraically closed in $F_{k}$. Moreover,

$$
d\left(Q_{k} \mid Q_{k-1}\right)=(m+1)(p-1) \text { where } Q_{k} \in \mathbb{P}\left(F_{k}\right) \text { lies above } Q_{k-1} .
$$


Now assuming that (ii) holds, we have that $g\left(F_{k}\right) \rightarrow \infty$ as $k \rightarrow \infty$. Now it follows from the construction of $F_{k}$, for $k \geq 0$, that the sequence $\mathcal{F} / \mathbb{F}_{q}=\left(F_{k} / \mathbb{F}_{q}\right)_{k \geq 0}$ is a tower with $\left[F_{k}: F_{k-1}\right]=p$ for all $k \geq 0$. Moreover, for all $i \geq 0$, by Kummer's Theorem [14], each place $P \in S_{i}$ splits completely in $F_{k}$, for any $k \geq i$. Thus, for all $i \geq 0$, we obtain that

$$
B_{n_{i}}\left(F_{k}\right) \geq\left[F_{k}: F_{i}\right]=p^{k-i} .
$$

(ii) We prove by induction. For $k=0$, it is trivial. The rest of the proof follows by using (60) and the Hurwitz Genus Formula.

Proposition 5.25. Let $\mathbb{F}_{q}$ be a finite field of characteristic $p$ and $m \in \mathbb{N}$ with $(m, p)=1$ and $m \geq 2$. Then there is a tower $\mathcal{F} / \mathbb{F}_{q}=\left(F_{k} / \mathbb{F}_{q}\right)_{k \geq 0}$ and a strictly increasing sequence $\left(n_{i}\right)_{i \geq 0}$ of positive integers with $n_{0}=1$ such that the steps of this tower $\mathcal{F} / \mathbb{F}_{q}=\left(F_{k} / \mathbb{F}_{q}\right)_{k \geq 0}$ have a class number such that

$$
h\left(F_{k} / \mathbb{F}_{q}\right) \geq h_{B R T 1, k}
$$

where

(61)

$$
\begin{array}{r}
h_{B R T 1, k}=\frac{(q-1)^{2}}{\left(g_{k}+1\right)(q+1)-p^{k}}\left(\prod_{i=0}^{k}\left(\begin{array}{c}
p^{k-i}+l_{i} \\
l_{i}
\end{array}\right)\right. \\
\left.+q^{g_{k}-1} \prod_{i=0}^{k}\left[\left(\frac{q^{n_{i}}}{q^{n_{i}}-1}\right)^{p^{k-i}}-p^{k-i}\left(\begin{array}{c}
p^{k-i}+m_{i} \\
p^{k-i}
\end{array}\right) \int_{0}^{\frac{1}{q^{n_{i}}}} \frac{\left(\frac{1}{q^{n_{i}}}-t\right)^{m_{i}}}{(1-t)^{p^{k-i}+m_{i}+1}} \mathrm{dt} .\right]\right) .
\end{array}
$$

where $g_{k}=\frac{m-1}{2}\left(p^{k}-1\right)$ and $\sum_{i=0}^{k} n_{i} m_{i} \leq g_{k}-2$ for all $k \geq 0$.

\section{Annexe}

The following table gives the best current bounds according to the known information on the number of places and the size of the genus. Recall that the bounds $h_{L M D}, h_{B R}, h_{A H L}$ and $h_{B R T}$ are respectively defined by (1), (2), (3) and (25).

\begin{tabular}{||c|l|l||}
\hline \hline Known information & case & reference \\
\hline \hline None specific information & & $h_{L M D}$ [11] \\
One degree $r$ with known $B_{r}$ & $r=1$ & $\begin{array}{l}h_{A H L} \text { [1] (low } g \\
\left.\text { and } B_{1} \geq(\sqrt{q}-1) g+1\right) \\
h_{B R}[5](\mathrm{else})\end{array}$ \\
& $r>1$ & $h_{B R T}$ \\
\hline Several degrees $r_{i}$ with known $B_{r_{i}}$ & & $h_{B R T}$ \\
\hline \hline
\end{tabular}

In case we only know the number of places of degree one, as noted previously $h_{B R}=h_{B R T}$ (see Remark 4.3). In this case, for very low values of $g$ and only if the number of rational points $B_{1}$ is such that $B_{1} \geq(\sqrt{q}-1) g+1$, bound $h_{A H L}$ is 
well suited else for almost values of $g$, bound $h_{B R}$ is better. In case we only know the number of places of degree $r>1$, the bound $h_{B R T}$ is better than $h_{B R}$. In case of several distinct degrees, bound $h_{B R T}$ gives the best results.

\section{ACKnowledgments}

Seher Tutdere is partially supported by TÜBİTAK under Grant No. TBAG$109 T 672$.

\section{REFERENCES}

[1] Yves Aubry, Safia Haloui, and Gilles Lachaud. Sur le nombre de points rationnels des variétés abéliennes et des jacobiennes sur les corps finis. C. R. Acad. Sci. Paris, Ser. I, 350:907-910, 2012.

[2] Yves Aubry, Safia Haloui, and Gilles Lachaud. On the number of points on abelian and jacobian varieties over finite fields. Acta Arithmetica, Accepted.

[3] Stéphane Ballet and Robert Rolland. Families of curves over any finite field attaining the generalized drinfeld-vladut bound. Publications Mathématiques de Besançon, Algèbre et Théorie des Nombres, pages 5-18, 2011.

[4] Stéphane Ballet and Robert Rolland. Minoration du nombre de classes des corps de fonctions algébriques définis sur un corps fini. C.R. Acad. Sci. Paris, Ser. I, 349(13-14):709-712, July 2011.

[5] Stéphane Ballet and Robert Rolland. Lower bounds on the class number of algebraic function fields defined over any finite field. Journal de théorie des nombres de Bordeaux, 24(3):505-540, 2012.

[6] Alp Bassa, Arnaldo Garcia, and Henning Stichtenoth. A new tower over cubic finite fields. Moscow Mathematical Journal, 8(3):401-418, 2008.

[7] Florian Hess, Henning Stichtenoth, and Seher Tutdere. On invariants of towers of function fields over finite fields. Journal of Algebra and Its Applications, 12(4):477-487, 2013.

[8] Arnaldo Garcia and Henning Stitchtenoth. A tower of artin-schreier extensions of function fields attaining the drinfeld-vladut bound. Inventiones Mathematicae, 121:211-222, 1995.

[9] Arnaldo Garcia, Henning Stitchtenoth, and Hans-Georg Ruck. On tame towers over finite fields. Journal für die reine und angewandte Mathematik, 557:53-80, 2003.

[10] Arnaldo Garcia, Henning Stitchtenoth, and Michael Thomas. On towers and composita of towers of function fields over finite fields. Finite Fields and their Applications, 3:257-274, 1997.

[11] Gilles Lachaud and Mireille Martin-Deschamps. Nombre de points des jacobiennes sur un corps finis. Acta Arithmetica, 56(4):329-340, 1990.

[12] Philippe Lebacque. Generalised mertens and brauer-siegel theorems. Acta Arithmetica, 130(4):333-350, 2007.

[13] Henning Stichtenoth. Algebraic Function Fields and Codes. Number 314 in Lectures Notes in Mathematics. Springer-Verlag, 1993.

[14] Henning Stichtenoth. Algebraic Function Fields and Codes. Number 254 in Graduate Texts in Mathematics. Springer-Verlag, second edition, 2008.

[15] Michael Tsfasman. Some remarks on the asymptotic number of points. In H. Stichtenoth and M.A. Tsfasman, editors, Coding Theory and Algebraic Geometry, volume 1518 of Lecture Notes in Mathematics, pages 178-192, Berlin, 1992. Springer-Verlag. Proceedings of AGCT3 conference, June 17-21, 1991, Luminy.

[16] Michael Tsfasman and Serguei Vladut. Asymptotic properties of zeta-functions. Journal of Mathematical Sciences, 84(5):1445-1467, 1997.

[17] Michael Tsfasman and Serguei Vladut. Infinite global fields and the generalized brauer-siegel theorem. Mosc. Math. J., 2(2):329-402, 2002.

[18] Michael Tsfasman, Serguei Vladut, and Dmitry Nogin. Algebraic Geometric Codes: Basic Notions, volume 139 of Mathematical Surveys and Monographs. American Mathematical Society, 2007.

[19] Seher Tutdere. On the asymptotic theory of towers of function fields over finite fields. $\mathrm{PhD}$ thesis, SabanciUniversity, 2012. 
[20] André Weil. Sur les courbes algébriques et les variétés qui s'en déduisent. Variétés abéliennes et courbes algébriques. Hermann, 1948. Publications de l'Institut de mathématiques de l'Université de Strasbourg, fasc. VII et VIII.

[21] André Weil. Basic Number Theory. Springer, 1967.

[22] Jörg Wulftange. Zahme Türme algebraischer Funktionenkörper. PhD thesis, Essen University, 2002 .

Aix-Marseille Université, Institut de Mathématiques de Luminy, case 930, F13288 Marseille Cedex 9, France

E-mail address: stephane.ballet@univ-amu.fr

Aix-Marseille Université, Institut de Mathématiques de Luminy, case 930, F13288 Marseille Cedex 9, France

E-mail address: robert.rolland@acrypta.fr

Orta DoĞu Teknik Üniversitesi, Uygulamali Matematik Enstitüsü, Kriptoloji Laboratuvari, Üniversiteler Mah. Dumlupinar Bul. No:1, 06800 Çankaya/Ankara, TURKEY

E-mail address: stutdere@gmail.com 